\title{
New Candidates Welcome! \\ Possible Winners with respect to the Addition of New Candidates
}

\author{
Y. Chevaleyre \\ LIPN
}

Univ. Paris 13
J. Lang

LAMSADE

Univ. Paris-Dauphine

J. Monnot

LAMSADE

Univ. Paris-Dauphine
N. Maudet

LIP6

Univ. Pierre et Marie Curie

L. Xia

SEAS

Harvard University

September 21, 2018

\begin{abstract}
In voting contexts, some new candidates may show up in the course of the process. In this case, we may want to determine which of the initial candidates are possible winners, given that a fixed number $k$ of new candidates will be added. We give a computational study of this problem, focusing on scoring rules, and we provide a formal comparison with related problems such as control via adding candidates or cloning.
\end{abstract}

\section{Introduction}

In many real-life collective decision making situations, the set of candidates (or alternatives) may vary while the voting process goes on, and may change at any time before the decision is final: some new candidates may join, whereas some others may withdraw. This, of course, does not apply to situations where the vote takes place in a very short period of time (such as, typically, political elections in most countries), and neither does the addition of new candidates during the process apply to situations where the law forbids new candidates to be introduced after the voting process has started (which, again, is the case for most political elections). However, there are quite many practical settings where this may happen, especially situations where votes are sent by email during an extended period of time. This is typically the case when making a decision about the date and time of a meeting. In the course of the process, we may learn that the room is taken at a given time slot, making this time slot no longer a candidate. The opposite case also occurs frequently; we thought the room was taken on a given date and then we learn that it has become available, making this time slot a new candidate.

The paper focuses on candidate addition only. More precisely, the class of situations we consider is the following. A set of voters have expressed their votes about a set of (initial) candidates. Then some new 
candidates declare their intention to participate in the election. The winner will ultimately be determined using some given voting rule and the voters' preferences over the set of all candidates. In this class of situations, an important question arises: who among the initial candidates can still be a winner once the voters' preferences about all candidates are known? This is important in particular if there is some interest to detect as soon as possible the candidates who are not possible winners: for instance, candidates for a job may have the opportunity to apply for different positions, and time slots may be released for other potential meetings.

This question is strongly related to several streams of work in the recent literature on computational social choice, especially the problem of determining whether the vote elicitation process can be terminated [10, 29]; the possible winner problem, and more generally the problem of applying a voting rule to incomplete preferences [22, 26, 30, 5, 6] or uncertain preferences with probabilistic information [20]; swap bribery, encompassing the possible winner problem as a particular case [15]; voting with an unknown set of available candidates [25]; the control of a voting rule by the chair via adding candidates; and resistance to cloning-we shall come back to the latter two problems in more detail in the related work section.

Clearly, considering situations where new voters are added is a specific case of voting under incomplete preferences, where incompleteness is of a very specific type: the set of candidates is partitioned in two groups (the initial and the new candidates), and the incomplete preferences consist of complete rankings on the initial candidates. This class of situations is, in a sense, dual to a class of situations that has been considered more often, namely, when the set of voters is partitioned in two groups: those voters who have already voted, and those who have not expressed their votes yet. The latter class of situations, while being a subclass of voting under incomplete preferences, has been more specifically studied as a coalitional manipulation problem [11,18], where the problem is to determine whether it is possible for the voters who have not voted yet to make a given candidate win. Varying sets of voters have also been studied in the context of compiling the votes of a subelectorate [8, 31]: there, one is interested in summarizing a set of initial votes, while still being able to compute the outcome once the remaining voters have expressed their votes.

The layout of the paper is as follows. In Section 2 we recall the necessary background on voting and we introduce some notation. In Section 3 we state the problem formally, by defining voting situations where candidates may be added after the votes over a subset of initial candidates have already been elicited. In the following sections we focus on specific voting rules and we study the problem from a computational point of view. In Section 4 we focus on the family of $K$-approval rules, including plurality and veto as specific subcases, and give a full dichotomy result for the complexity of the possible winner problem with respect to the addition of $k$ new candidates; namely, we show that the problem is NP-complete as soon as $K \geq 3$ and $k \geq 3$, and polynomial if $K \leq 2$ or $k \leq 2$. In Section 5 we focus on the Borda rule and show that the problem is polynomial-time solvable regardless of the number of new candidates. We also exhibit a more general family of voting rules, including Borda, for which this result can be generalized. In Section 6 we show that the problem can be hard for some positional scoring rules even if only one new candidate is added. In Section 7 we discuss the relationship to the general possible winner problem, to the control of an election by the chair via adding candidates, and to candidate cloning. Section 8 summarizes the results and mentions further research directions. 


\section{Background and notation}

Let $C$ be a finite set of candidates, and $N$ a finite set of voters. The number of voters is denoted by $n$, and the (total) number of candidates by $m$. A $C$-vote (called simply a vote when this is not ambiguous) is a linear order over $C$, denoted by $\succ$ or by $V$. We sometimes denote votes in the following way: $a \succ b \succ c$ is denoted by $a b c$, etc. An $n$-voter $C$-profile is a collection $P=\left\langle V_{1}, \ldots, V_{n}\right\rangle$ of $C$-votes. Let $\mathcal{P}_{C}$ be the set of all $C$-votes and therefore $\mathcal{P}_{C}^{n}$ be the set of all $n$-voter $C$-profiles. We denote by $\mathcal{P}_{C}^{*}$ the set of all $n$-voter $C$-profiles for $n \geq 1$, i.e., $\mathcal{P}_{C}^{*}=\cup_{n \geq 1} \mathcal{P}_{C}^{n}$.

A voting rule on $C$ is a function $r$ from $\mathcal{P}_{C}^{*}$ to $C$. A voting correspondence is a function from $\mathcal{P}_{C}^{*}$ to $2^{C} \backslash\{\emptyset\}$. The most natural way of obtaining a voting rule from a voting correspondence is to break ties according to a fixed priority order on candidates. In this paper, we do not fix a priority order on candidates (one reason being that the complete set of candidates is not known to start with), which means that we consider voting correspondences rather than rules, and ask whether $x$ is a possible cowinner for a given profile $P$. This is equivalent to asking whether there exists a priority order for which $x$ is a possible winner, or else whether $x$ is a possible winner for the most favorable priority order (with $x$ having priority over all other candidates). This is justified in our context by the fact that specifying such a priority order is problematic when we don't know in advance the identities of the potential new candidates. With a slight abuse of notation we denote voting correspondences by $r$ just as voting rules. Let $r(P)$ be the set of cowinners for profile $P$.

For $P \in \mathcal{P}_{C}^{*}$ and $x, x^{\prime} \in C$, let $n(P, i, x)$ be the number of votes in $P$ ranking $x$ in position $i$, ntop $(P, x)=$ $n(P, 1, x)$ the number of votes in $P$ ranking $x$ first, and $N_{P}\left(x, x^{\prime}\right)$ the number of votes in $P$ ranking $x$ above $x^{\prime}$. Let $\vec{s}=\left\langle s_{1}, \ldots, s_{m}\right\rangle$ be a vector of integers such that $s_{1} \geq \ldots \geq s_{m}$ and $s_{1}>s_{m}$. The scoring rule $r_{\vec{s}}(P)$ induced by $\vec{s}$ elects the candidate(s) maximizing $S_{\vec{s}}(x, P)=\sum_{i=1}^{m} s_{i} \cdot n(P, i, x)$.

If $K$ is a fixed integer then $K$-approval, $r_{K}$, is the scoring rule corresponding to the vector $\overrightarrow{s_{K}}=$ $\langle 1, \ldots, 1,0, \ldots, 0\rangle-$ with $K 1$ 's and $m-K 0$ 's. The $K$-approval score $S_{s_{K}}(x, P)$ of a candidate $x$ is denoted more simply by $S_{K}(x, P)$ : in other words, $S_{K}(x, P)$ is the number of voters in $P$ who rank $x$ in the first $K$ positions, i.e., $S_{K}(x, P)=\sum_{i=1, \ldots, K} n(P, i, x)$. When $K=1$, we get the plurality rule $r_{P}$, and when $K=m-1$ we get the veto (or antiplurality) rule. The Borda rule $r_{B}$ is the scoring rule corresponding to the vector $\langle m-1, m-2, \ldots, 0\rangle$.

We now define formally situations where new candidates are added.

Definition $1 A$ voting situation with a varying set of candidates is a 4-tuple $\Sigma=\left\langle N, X, P_{X}, k\right\rangle$ where $N$ is a set of voters (with $|N|=n$ ), $X$ a set of candidates, $P_{X}=\left\langle V_{1}, \ldots, V_{n}\right\rangle$ an n-voter $X$-profile, and $k$ is a positive integer, encoded in unary.

$X$ denotes the set of initial candidates, $P_{X}$ the initial profile, and $k$ the number of new candidates. Nothing is known a priori about the voters' preferences over the new candidates, henceforth their identity is irrelevant and only their number counts. The assumption that $k$ is encoded in unary ensures that the number of new candidates is polynomial in the size of the input. Most of our results would still hold if the number of new candidates is exponentially large in the size of the input, but for the sake of simplicity, and also because, in practice, $k$ will be small anyway, we prefer to exclude this possibility.

Because the number of candidates is not the same before and after the new candidates come in, we have to consider families of voting rules (for a varying number of candidates) rather than voting rules for a fixed number of candidates. While it is true that for many usual voting rules there is an obvious way of defining them for a varying number of candidates, this is not the case for all of them, especially scoring rules. Still, some 
natural scoring rules, including plurality, veto, more generally $K$-approval, as well as Borda, are naturally defined for any number of candidates. We shall therefore consider families of voting rules, parameterized by the number of candidates $\left(r^{m}\right)$. We slightly abuse notation and denote these families of voting rules by $r$, and consequently often write $r(P)$ instead of $r^{m}(P)$. The complexity results we give in this paper make use of such families of voting rules, where the number of candidates is variable.

If $P$ is a $C$-profile and $C^{\prime} \subseteq C$, then the projection of $P$ on $C^{\prime}$, denoted by $P^{\downarrow} C^{\prime}$, is obtained by deleting all candidates in $C \backslash C^{\prime}$ in each of the votes of $P$, and leaving unchanged the ranking on the candidates of $C^{\prime}$. For instance, if $P=\langle a b c d, d c a b\rangle$, then $P^{\downarrow\{a, b\}}=\langle a b, a b\rangle$ and $P^{\downarrow\{a, b, c\}}=\langle a b c, c a b\rangle$. In all situations, the set of initial candidates is denoted by $X=\left\{x_{1}, \ldots, x_{p}\right\} \cup\left\{x^{*}\right\}$, the set of the $k$ new candidates is denoted by $Y=\left\{y_{1}, \ldots, y_{k}\right\}$. If $P_{X}$ is an $X$-profile and $P^{\prime}$ an $X \cup Y$-profile, then we say that $P^{\prime}$ extends $P_{X}$ if the projection of $P^{\prime}$ on $X$ is exactly $P_{X}$. For instance, let $X=\left\{x_{1}, x_{2}, x_{3}\right\} \cup\left\{x^{*}\right\}$, $Y=\left\{y_{1}, y_{2}\right\}$; the profile $P^{\prime}=\left\langle x_{1} y_{1} x^{*} x_{2} y_{2} x_{3}, y_{1} y_{2} x_{1} x_{2} x_{3} x^{*}, x_{3} x_{2} y_{2} x^{*} y_{1} x_{1}\right\rangle$ extends the $X$-profile $P_{X}=$ $\left\langle x_{1} x^{*} x_{2} x_{3}, x_{1} x_{2} x_{3} x^{*}, x_{3} x_{2} x^{*} x_{1}\right\rangle$.

\section{Possible winners when new candidates are added}

We recall from [22] that given a collection $\left\langle P_{1}, \ldots, P_{n}\right\rangle$ of partial strict orders on $C$ representing some incomplete information about the votes, a candidate $x^{*}$ is a possible winner if there is a profile $\left\langle T_{1}, \ldots, T_{n}\right\rangle$ where each $T_{i}$ is a ranking on $C$ extending $P_{i}$ in which $x^{*}$ wins. Reformulated for the case where $P_{i}$ is a ranking of the initial candidates (those in $X$ ), we get the following definition:

Definition 2 Given a voting situation $\Sigma=\left\langle N, X, P_{X}, k\right\rangle$, and a collection $r$ of voting rules, we say that $x^{*} \in X$ is a possible cowinner with respect to $\Sigma$ and $r$ if there is a $(X \cup Y)$-profile $P^{\prime}$ extending $P_{X}$ such that $x^{*} \in r\left(P^{\prime}\right)$, where $Y=\left\{y_{1}, \ldots, y_{k}\right\}$ is a set of $k$ new candidates.

Note that we do not have $Y$ in the input, because it would be redundant with $k$ : it is enough to know the number of new candidates. Note also that all new candidates $\left\{y_{1}, \ldots, y_{k}\right\}$ have to appear in the extended votes composing $P^{\prime}$.

Also, we do not consider the problem of deciding whether a new candidate $y_{j}$ is a possible cowinner, because it is trivial. Indeed, as soon as the voting correspondence satisfies the extremely weak property that a candidate ranked first by all voters is always a cowinner (which is obviously satisfied by all common voting rules), any new candidate is a possible cowinner.

We now define formally the problems we study in this paper.

Definition 3 Given a collection $r$ of voting rules, the POSSIBLE COWINNER PROBLEM WITH NEW CANDIDATES (or PCWNC) for $r$ is defined as follows:

Input $A$ voting situation $\Sigma=\langle N, X, P, k\rangle$ and a candidate $x^{*} \in X$.

Question Is $x^{*}$ a possible cowinner with respect to $\Sigma$ and $r$ ?

Also, the subproblem of $\mathrm{PCWNC}$ where the number $k$ of new candidates is fixed will be denoted by $\mathrm{PCWNC}(k)$.

We can also define the notion of necessary cowinner with respect to $\Sigma$ and $r: x^{*} \in X$ is a necessary cowinner with respect to $\Sigma, Y$, and $r$ if for every $(X \cup Y)$-profile $P^{\prime}$ extending $P_{X}$ we have $x^{*} \in r(P)$. 
However, the study of necessary cowinners in this particular setting will almost never lead to any significant results. There may be necessary cowinners among the initial candidates, but this will happen rarely (and this case will be discussed for a few specific voting rules in the corresponding parts of the paper).

Now we are in position to consider specific voting rules.

\section{$4 \quad K$-approval}

As a warm-up we start by considering the plurality rule.

\subsection{Plurality}

Let us start with an example: suppose $X=\{a, b, c\}, n=13$, and the plurality scores in $P_{X}$ are $a \mapsto 6, b \mapsto 4$, $c \mapsto 3$. There is only one new candidate $(y)$. We have:

1. $a$ is a possible cowinner ( $a$ will win in particular if the top candidate of every voter remains the same);

2. $b$ is a possible cowinner: to see this, suppose that 2 voters who had ranked $a$ first now rank $y$ first; the new scores are $a \mapsto 4, b \mapsto 4, c \mapsto 3, y \mapsto 2$;

3. $c$ is not a possible cowinner: to reduce the scores of $a$ (resp. $b$ ) to that of $c$, we need at least 3 (resp. 1) voters who had ranked $a$ (resp. $b$ ) first to now rank $y$ first; but this then means that $y$ gets at least 4 votes, while $c$ has only 3 .

More generally, we have the following result:

Proposition 1 Let $P_{X}$ be an $n$-voter profile on $X$, and $x^{*} \in X$. The candidate $x^{*}$ is a possible cowinner for $P_{X}$ and plurality with respect to the addition of $k$ new candidates if and only if

$$
n \operatorname{nop}\left(P_{X}, x^{*}\right) \geq \frac{1}{k} \cdot \sum_{x_{i} \in X} \max \left(0, n t o p\left(P_{X}, x_{i}\right)-\operatorname{ntop}\left(P_{X}, x^{*}\right)\right)
$$

Proof: Suppose first that the inequality holds. We build the following $(X \cup Y)$-profile $P^{\prime}$ extending $P_{X}$ :

1. for every candidate $x_{i}$ such that $n \operatorname{top}\left(P_{X}, x_{i}\right)>\operatorname{ntop}\left(P_{X}, x^{*}\right)$ we simply take ntop $\left(P_{X}, x_{i}\right)-n$ top $\left(P_{X}, x^{*}\right)$ arbitrary votes ranking $x_{i}$ on top and place one of the $y_{j}$ 's on top of the vote (and the other $y_{j}$ 's anywhere), subject to the condition that no $y_{j}$ is placed on top of a vote more than $n t o p\left(P_{X}, x^{*}\right)$ times. (This is possible because the inequality is satisfied).

2. in all other votes (those not considered at step 1), place all $y_{j}$ 's anywhere except on top.

We obtain a profile $P^{\prime}$ extending $P_{X}$. First, we have $n t o p\left(P^{\prime}, x^{*}\right)=n t o p\left(P_{X}, x^{*}\right)$, because in all the votes in $P_{X}$ where $x^{*}$ is on top, the new top candidate in the corresponding vote in $P^{\prime}$ is still $x^{*}$ (cf. step 2), and all the votes in $P_{X}$ where $x^{*}$ was not on top obviously cannot have $x^{*}$ on top in the corresponding vote in $P^{\prime}$. Second, let $x_{i} \neq x^{*}$. If $n \operatorname{top}\left(P_{X}, x_{i}\right) \leq n \operatorname{nop}\left(P_{X}, x^{*}\right)$ then $n \operatorname{top}\left(P^{\prime}, x_{i}\right)=n \operatorname{top}\left(P_{X}, x_{i}\right)$; and if $n$ top $\left(P_{X}, x_{i}\right)>$ $n$ top $\left(P_{X}, x^{*}\right)$ then we have $n t o p\left(P^{\prime}, x_{i}\right)=n \operatorname{top}\left(P_{X}, x_{i}\right)-\left(n t o p\left(P_{X}, x_{i}\right)-n \operatorname{top}\left(P_{X}, x^{*}\right)\right)=n t o p\left(P_{X}, x^{*}\right)$. Therefore, $x^{*}$ is a cowinner for plurality in $P^{\prime}$.

Conversely, if the inequality is not satisfied, in order for $x^{*}$ to become a cowinner in $P^{\prime}$, the other $x_{i}$ 's 
must lose globally an amount of $\sum_{x_{i} \in X} \max \left(0, n t o p\left(P_{X}, x_{i}\right)-n t o p\left(P_{X}, x^{*}\right)\right)$ votes. But since we have $\sum_{x_{i} \in X} \max \left(0, n \operatorname{nop}\left(P_{X}, x_{i}\right)-n \operatorname{top}\left(P_{X}, x^{*}\right)\right)>k \cdot n \operatorname{ntop}\left(P_{X}, x^{*}\right)$, for at least one of the $y_{j}$ 's it must hold that $n \operatorname{top}\left(P^{\prime}, y_{j}\right)>n \operatorname{top}\left(P^{\prime}, x^{*}\right)$; therefore $x^{*}$ cannot be a cowinner for plurality in $P^{\prime}$.

We do not need to pay much attention to the veto rule, since the characterization of possible cowinners is trivial. Indeed, by placing any of the new candidates below $x^{*}$ in every vote of $P_{X}$ where $x^{*}$ is ranked at the bottom position, we obtain a vote $P^{\prime}$ where no one vetoes $x^{*}$, so any candidate is a possible cowinner.

As a corollary, computing possible cowinners for the rules of plurality (and veto) with respect to candidate addition can be computed in polynomial time (which we already knew, since possible cowinners for plurality and veto can be computed in polynomial time [5]).

\section{2 $K$-approval, one new candidate}

We start with the case where a single candidate is added. Recall that we denote by $S_{K}\left(x_{j}, P_{X}\right)$ the score of $x_{j}$ for $P_{X}$ and $K$-approval (i.e. the number of voters who rank $x_{j}$ among their top $K$ candidates); and by $n\left(P_{X}, K, x_{j}\right)$ the number of voters who rank $x_{j}$ exactly in position $K$.

Proposition 2 Let $K$ be an positive integer, $P_{X}$ be an $n$-voter profile on $X$, and $x^{*} \in X$. The candidate $x^{*}$ is a possible cowinner for $P_{X}$ and $K$-approval with respect to the addition of one new candidate if and only if the following two conditions hold:

$$
\begin{aligned}
& \text { 1. for every } x_{i} \neq x^{*} \text {, if } S_{K}\left(x_{i}, P_{X}\right)>S_{K}\left(x^{*}, P_{X}\right) \\
& \text { then } n\left(P_{X}, K, x_{i}\right) \geq S_{K}\left(x_{i}, P_{X}\right)-S_{K}\left(x^{*}, P_{X}\right) \text {. } \\
& \text { 2. } S_{K}\left(x^{*}, P_{X}\right) \geq \sum_{x_{i} \in X} \max \left(0, S_{K}\left(x_{i}, P_{X}\right)-S_{K}\left(x^{*}, P_{X}\right)\right)
\end{aligned}
$$

Proof: Assume conditions (1) and (2) are satisfied. Then, we build the following $(X \cup\{y\})$-profile extending $P_{X}$ :

(i) for every $x_{i}$ such that $S_{K}\left(x_{i}, P_{X}\right)>S_{K}\left(x^{*}, P_{X}\right)$, we take $S_{K}\left(x_{i}, P_{X}\right)-S_{K}\left(x^{*}, P_{X}\right)$ arbitrary votes who rank $x_{i}$ in position $K$ in $P_{X}$ and place $y$ on top (condition (1) ensures that we can find enough such votes).

(ii) in all other votes (those not considered at step (i)), place $y$ in the bottom position.

We obtain a profile $P^{\prime}$ extending $P_{X}$. First, we have $S_{K}\left(x^{*}, P^{\prime}\right)=S_{K}\left(x^{*}, P_{X}\right)$, because (a) all votes in $P_{X}$ ranking $x^{*}$ in position $K$ are extended in such a way that $y$ is placed in the bottom position, therefore $x^{*}$ gets a point in each of these votes if and only if it got a point in $P_{X}$, and (b) in all the other votes (those where $x^{*}$ is not ranked in position $K$ in $P_{X}$ ), $x^{*}$ certainly gets a point in $P^{\prime}$ if and only if they got a point in $P_{X}$. This holds both in the case where $y$ was added at the top or the bottom of the vote. Second, for every $x_{i}$ such that $S_{K}\left(x_{i}, P_{X}\right)>S_{K}\left(x^{*}, P_{X}\right), x_{i}$ loses exactly $S_{K}\left(x_{i}, P_{X}\right)-S_{K}\left(x^{*}, P_{X}\right)$ points when $P_{X}$ is extended into $P^{\prime}$, therefore $S_{K}\left(x_{i}, P^{\prime}\right)=S_{K}\left(x_{i}, P_{X}\right)-S_{K}\left(x_{i}, P_{X}\right)+S_{K}\left(x^{*}, P_{X}\right)=S_{K}\left(x^{*}, P_{X}\right)$. Third, $S_{K}\left(y, P^{\prime}\right)=\sum_{x_{i} \in X} \max \left(0, S_{K}\left(x_{i}, P_{X}\right)-S_{K}\left(x^{*}, P_{X}\right)\right) \leq S_{K}\left(x^{*}, P_{X}\right)$-because of (2)-hence $S_{K}\left(y, P^{\prime}\right) \leq S_{K}\left(x^{*}, P^{\prime}\right)$. Therefore, $x^{*}$ is a cowinner for $K$-approval in $P^{\prime}$. 
Now, assume condition (1) is not satisfied, that is, there is an $x_{i}$ such that $S_{K}\left(x_{i}, P_{X}\right)>S_{K}\left(x^{*}, P_{X}\right)$ and such that $n\left(P_{X}, K, x_{i}\right)<S_{K}\left(x_{i}, P_{X}\right)-S_{K}\left(x^{*}, P_{X}\right)$. There is no way of having $x_{i}$ lose more than $S_{K}\left(x_{i}, P_{X}\right)$ points, therefore $x^{*}$ will never catch up with $x_{i}$ 's advantage and is therefore not a possible cowinner. Finally, assume condition (2) is not satisfied, which means that we have $\sum_{x_{i} \in X} \max \left(0, S_{K}\left(x_{i}, P_{X}\right)-\right.$ $\left.S_{K}\left(x^{*}, P_{X}\right)\right)>S_{K}\left(x^{*}, P_{X}\right)$. Then, in order for $x^{*}$ to reach the score of $x_{i}$ 's we must add $y$ in one of the top $K$ positions in a number of votes exceeding $S_{K}\left(x^{*}, P_{X}\right)$, therefore $S_{K}\left(y, P^{\prime}\right)>S_{K}\left(x^{*}, P_{X}\right) \geq S_{K}\left(x^{*}, P^{\prime}\right)$, and therefore $x^{*}$ is not a possible cowinner.

Therefore, computing possible cowinners for $K$-approval with respect to the addition of one candidate can be done in polynomial time.

\subsection{2-approval, any (fixed) number of new candidates}

For each profile $P$ and each candidate $x^{\prime}$, we simply write $s\left(x^{\prime}, P\right)$ for the score of $x^{\prime}$ in $P$ under $r_{2}$, that is, $s\left(x^{\prime}, P\right)=S_{2}\left(x^{\prime}, P\right)$, i.e. the number of times that $x^{\prime}$ is ranked within the top two positions in $P$.

Let $P_{X}=\left\langle V_{1}, \ldots, V_{n}\right\rangle$ be an initial profile and $Y=\left\{y_{1}, \ldots, y_{k}\right\}$ the set of new candidates. Let $x^{*} \in X$. We want to know whether $x^{*}$ is a possible cowinner for 2-approval and $P_{X}$. Let us partition $P_{X}$ into $P_{1}, P_{2}$ and $P_{3}$, where $P_{1}$ consists of the votes in which $x^{*}$ is ranked in the top position, $P_{2}$ consists of the votes in which $x^{*}$ is ranked in the second position and $P_{3}$ consists of the votes in which $x^{*}$ is not ranked within the top two positions. Let $P$ be an extension of $P_{X}$ to $X \cup Y$. For each candidate $x^{\prime} \in X$, we define the following three subsets of $P$ :

- $\operatorname{HP}\left(P, x^{\prime}\right)$ is the set of votes in $P$ where $x^{\prime}$ is ranked in the second position and neither $x^{*}$ nor any new candidate is ranked in the top position (HP stands for "high priority").

- $\operatorname{MP}\left(P, x^{\prime}\right)$ is the set of votes in $P$ where $x^{*}$ or any new candidate is ranked in the top position and $x^{\prime}$ is ranked in the second position (MP stands for "medium priority").

- $\operatorname{LP}\left(P, x^{\prime}\right)$ is the set of votes in $P$ where $x^{\prime}$ is ranked in the top position and some $x^{\prime \prime} \in X \backslash\left\{x^{*}\right\}$ is ranked in the second position (LP stands for "low priority").

These definitions also apply to $P_{X}$; our definitions then simplify into: $\operatorname{HP}\left(P_{X}, x^{\prime}\right)$ is the set of votes in $P_{X}$ where $x^{\prime}$ is ranked second and $x^{*}$ is not ranked first; $\operatorname{MP}\left(P_{X}, x^{\prime}\right)$ is the set of votes in $P_{X}$ where $x^{*}$ is ranked first and $x^{\prime}$ is ranked second; $\operatorname{LP}\left(P_{X}, x^{\prime}\right)$ is the set of votes in $P_{X}$ where $x^{\prime}$ is ranked first and $x^{*}$ is not ranked second. These definitions are summarized in Figure 1 Finally, for $x \in X \cup Y$, let $\Delta(P, x)=$ $S_{2}(x, P)-S_{2}\left(x^{*}, P\right)$.

Let us compute these sets on a concrete example, which will be reused throughout the section.

Example 1 Let $X=\left\{x^{*}, x_{1}, \ldots, x_{6}\right\}$ and consider the following profile $P_{X}$ consisting of 19 votes (we only mention the first two candidates in each vote):

\begin{tabular}{lllllllllllllllllll}
$v_{1}$ & $v_{2}$ & $v_{3}$ & $v_{4}$ & $v_{5}$ & $v_{6}$ & $v_{7}$ & $v_{8}$ & $v_{9}$ & $v_{10}$ & $v_{11}$ & $v_{12}$ & $v_{13}$ & $v_{14}$ & $v_{15}$ & $v_{16}$ & $v_{17}$ & $v_{18}$ & $v_{19}$ \\
\hline$x^{*}$ & $x_{1}$ & $x_{2}$ & $x_{3}$ & $x_{1}$ & $x_{1}$ & $x_{1}$ & $x_{2}$ & $x_{2}$ & $x_{2}$ & $x_{2}$ & $x_{2}$ & $x_{3}$ & $x_{3}$ & $x_{3}$ & $x_{3}$ & $x_{3}$ & $x_{3}$ & $x_{4}$ \\
$x_{1}$ & $x^{*}$ & $x^{*}$ & $x^{*}$ & $x_{4}$ & $x_{4}$ & $x_{5}$ & $x_{1}$ & $x_{3}$ & $x_{4}$ & $x_{5}$ & $x_{5}$ & $x_{1}$ & $x_{2}$ & $x_{4}$ & $x_{4}$ & $x_{5}$ & $x_{6}$ & $x_{6}$
\end{tabular}




\begin{tabular}{|c|c|c|}
\hline & top candidate belongs to & $2^{\text {nd }}$ candidate belongs to \\
\hline $\operatorname{HP}\left(P, x^{\prime}\right)$ & $X \backslash\left\{x^{*}\right\}$ & $\left\{x^{\prime}\right\}$ \\
\hline $\operatorname{MP}\left(P, x^{\prime}\right)$ & $Y \cup\left\{x^{*}\right\}$ & $\left\{x^{\prime}\right\}$ \\
\hline $\operatorname{LP}\left(P, x^{\prime}\right)$ & $\left\{x^{\prime}\right\}$ & $X \backslash\left\{x^{*}\right\}$ \\
\hline
\end{tabular}

Figure 1: A vote $V \in P$ belongs respectively to the sets $\operatorname{HP}(),. \operatorname{MP}(),. \operatorname{LP}($.$) if its top two candidates belong$ to the respective sets.

We have $P_{1}=\left\{v_{1}\right\}, P_{2}=\left\{v_{2}, v_{3}, v_{4}\right\}$ and $P_{3}=\left\{v_{5}, \ldots, v_{19}\right\}$. This is summarized together with the priority classification in the following table:

\begin{tabular}{|l|l|c|l|c|}
\hline & HP & MP & LP & $\Delta\left(P_{X}, x_{i}\right)$ \\
\hline$x_{1}$ & $v_{8}, v_{13}$ & $v_{1}$ & $v_{5}, v_{6}, v_{7}$ & 3 \\
$x_{2}$ & $v_{14}$ & & $v_{8}, v_{9}, v_{10}, v_{11}, v_{12}$ & 3 \\
$x_{3}$ & $v_{9}$ & & $v_{13}, v_{14}, v_{15}, v_{16}, v_{17}, v_{18}$ & 4 \\
$x_{4}$ & $v_{5}, v_{6}, v_{10}, v_{15}, v_{16}$ & & $v_{19}$ & 2 \\
$x_{5}$ & $v_{7}, v_{11}, v_{12}, v_{17}$ & & & 0 \\
$x_{6}$ & $v_{18}, v_{19}$ & & & -2 \\
\hline
\end{tabular}

If $P^{*}$ is an extension of $P_{X}$ to $X \cup Y$ then we write $P^{*}=\left\langle V_{1}^{*}, \ldots, V_{n}^{*}\right\rangle$, where $V_{i}^{*}$ is the vote over $X \cup Y$ extending $V_{i}$. We now establish a useful property of the extensions of $P_{X}$ for which $x^{*}$ is a cowinner. Without loss of generality, we assume that in every vote $V_{i}^{*}$, every new candidate $y_{j}$ is ranked either in the first two positions, or below all candidates of $X$.

Proposition 3 If there exists an extension $P$ of $P_{X}$ such that $x^{*} \in r_{2}(P)$, then there exists an extension $P^{*}$ of $P_{X}$ such that $x^{*} \in r_{2}\left(P^{*}\right)$, and satisfying the following conditions:

1. For each $V_{i} \in P_{X}$, if $x^{*}$ is ranked within the top two positions in $V_{i}$, then $x^{*}$ is also ranked within the top two positions in $V_{i}^{*}$.

2. For each $V_{i}^{*} \in P^{*}$, if the top candidate of $V_{i}^{*}$ is not in $Y$ then the second-ranked candidate of $V_{i}^{*}$ is not in $Y$ either.

3. For each $x^{\prime} \in X \backslash\left\{x^{*}\right\}$ and each $V_{i} \in \operatorname{MP}\left(P_{X}, x^{\prime}\right) \cup \operatorname{LP}\left(P_{X}, x^{\prime}\right)$, if $x^{\prime}$ is not ranked within the top two positions in $V_{i}^{*}$, then for each $V_{j} \in \operatorname{HP}\left(P_{X}, x^{\prime}\right)$, $x^{\prime}$ is not ranked within the top two positions in $V_{j}^{*}$.

Proof: We consider in turn the different conditions:

1. This is because if there exists $V^{\prime} \in P$ such that $x^{*}$ is not in the top two positions whereas $x^{*}$ is in the top two positions in its original vote $V \in P_{X}$, then we can simply move all of candidates in $Y$ ranked higher than $x^{*}$ to the bottom positions. Let $V^{*}$ denote the vote obtained this way. By replacing $V^{\prime}$ with $V^{*}$, we increase the score of $x^{*}$ by 1 , and the score of each other candidate by no more than 1 , which means that $x^{*}$ is still a cowinner.

2. If there exists $V^{\prime} \in P$ such that $x^{\prime} \in X$ is ranked in the top position and $y \in Y$ is ranked in the second position, then we simply obtain $V^{*}$ by switching $y$ and $x^{\prime}$. 
3. The condition states that for each candidate $x^{\prime}$, whenever we want to reduce its score, we should first try to reduce it by putting a new candidate $y \in Y$ on top of some vote in $V \in \operatorname{HP}\left(P_{X}, x^{\prime}\right)$. This is because by putting $y$ on top of some vote in $\operatorname{HP}\left(P_{X}, x^{\prime}\right)$, we may use only one extra candidate $y^{\prime} \in Y$ to reduce by one unit the score of the candidate ranked at the top position of $V$. Formally, suppose there exist $V_{1} \in \operatorname{HP}\left(P_{X}, x^{\prime}\right)$ and $V_{2} \in \operatorname{MP}\left(P_{X}, x^{\prime}\right) \cup \operatorname{LP}\left(P_{X}, x^{\prime}\right)$ such that $x^{\prime}$ is within the top two positions of $V_{1}^{\prime}$ (the extension of $V_{1}$ ) but not within the top two positions of $V_{2}^{\prime}$ (the extension of $V_{2}$ ). Let $y \in Y$ be any candidate ranked within the top two positions of $V_{2}^{\prime}$. Let $V_{2}^{*}$ denote the vote obtained from $V_{2}^{\prime}$ by moving $y$ to the bottom, and let $V_{1}^{*}$ denote the vote obtained from $V_{1}^{\prime}$ by moving $y$ to the top position. Next, we replace $V_{1}^{\prime}$ and $V_{2}^{\prime}$ by $V_{1}^{*}$ and $V_{2}^{*}$, respectively. It follows that the score of each candidate does not change, which means that $x^{*}$ is still a cowinner. We repeat this procedure until statement (3) is satisfied for every $x^{\prime} \in X \backslash\left\{x^{*}\right\}$. Since after each iteration there is at least one additional vote that will never be modified again, this procedure ends in $O\left(\left|P_{X}\right|\right)$ times.

Proposition 3 simply tells us that when looking for an extension that makes $x^{*}$ a cowinner, it suffices to restrict our attention to the extensions that satisfy conditions (1) to (3). Moreover, using (1) of Proposition 3 . we deduce that $s\left(x^{*}, P^{*}\right)=s\left(x^{*}, P_{X}\right)$. Hence, for votes $V \in P_{2}$ (the votes in which $x^{*}$ is ranked in the second position), we can assume that the new candidates of $Y$ are put in bottom positions in $P^{*}$.

Define $X^{\bullet}$ as the set of all candidates in $X$ such that $\Delta\left(P_{X}, x_{i}\right)>0$. Our objective is to reduce all score differences to 0 for $x \in X^{\bullet}$, while keeping the score differences of each new candidate non-positive. (We do not have to care about the candidates in $X \backslash X^{\bullet}$ ).

The intuition underlying our algorithm is that when trying to reduce $\Delta\left(P, x_{i}\right)$ on the current profile $P$, we first try to use the votes in $\operatorname{HP}\left(P_{X}, x_{i}\right)$, then the votes in $\operatorname{MP}\left(P_{X}, x_{i}\right)$, and finally the votes in $\operatorname{LP}\left(P_{X}, x_{i}\right)$. This is because putting some candidates from $Y$ in the top positions in the votes of $\operatorname{HP}\left(P_{X}, x_{i}\right)$ not only reduces $\Delta\left(P, x_{i}\right)$ by one unit, but also creates an opportunity to "pay" one extra candidate from $Y$ to reduce $\Delta\left(P, x_{j}\right)$ by one unit, where $x_{j}$ is the candidate ranked on top of this vote. For the votes in $\operatorname{MP}\left(P_{X}, x_{i}\right)$, we can only reduce $\Delta\left(P_{X}, x_{i}\right)$ by one unit without any other benefit. For the votes in $\operatorname{LP}\left(P_{X}, x_{i}\right)$ we will have to use two candidates from $Y$ to bring down $\Delta\left(P, x_{i}\right)$ by one unit; however, if we already put some $y \in Y$ in the top position in order to reduce $\Delta\left(P, x_{j}\right)$, where $x_{j}$ is the candidate ranked in the second position in the original vote, then we only need to pay one extra candidate in $Y$ to reduce $\Delta\left(P, x_{i}\right)$ by one unit. Therefore, the major issue consists in finding the most efficient way to choose the votes in $\operatorname{HP}\left(P_{X}, x_{i}\right)$ to reduce $\Delta\left(P, x_{i}\right)$, when $\Delta\left(P, x_{i}\right) \leq\left|\operatorname{HP}\left(P, x_{i}\right)\right|$. We will solve this problem by reducing it to a max-flow problem.

The algorithm is composed of a main function CheckCowinner(.) which comes together with two subfunctions AddNewAlternativeOnTop(.) and BuildMaxFlowGraph(.) that we detail first.

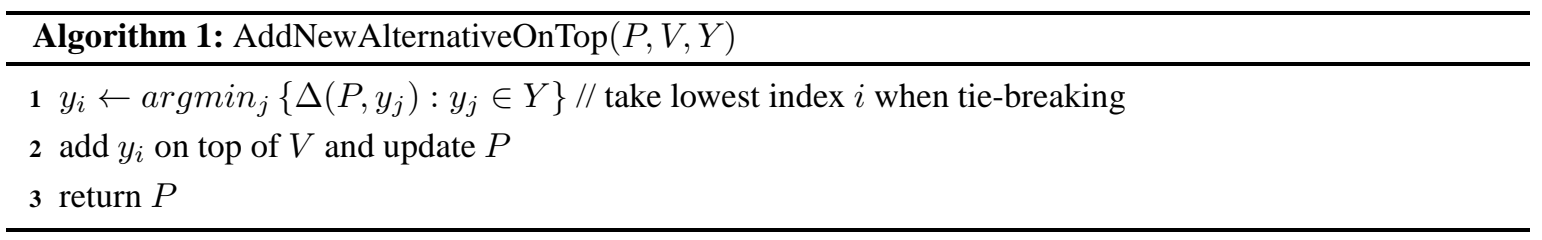

The procedure AddNewAlternativeOnTop simply picks new candidates to be put on top of votes, and up- 
dates subsequently the profile. Note that in this procedure, candidates from $Y$ to be added on top of the votes are those with the lowest score (or the lowest index, in case of ties). This results in choosing new candidates in a cyclic order $y_{1} \rightarrow y_{2} \ldots \rightarrow y_{|Y|} \rightarrow y_{1} \ldots$

As for the function BuildMaxFlowGraph $\left(P, x^{*}, X_{1}, X_{2}\right)$, it builds the weighted directed graph $G=$ $\langle W, E\rangle$ defined as follows:

- $W=\{s, t\} \cup X_{1} \cup X_{2} \cup \bigcup_{x_{i} \in X_{2}} \operatorname{LP}\left(P, x_{i}\right)$;

- $E$ contains the following weighted edges:

- for each $x \in X_{1}$, an edge $(s, x)$ with weight $\Delta(P, x)$;

- for each $x \in X_{2}$ and each $V \in \operatorname{LP}(P, x)$ : an edge $(V, x)$ with weight 1; plus, if the candidate $x^{\prime}$ in second position in $V$ is in $X_{1}$, an edge $\left(x^{\prime}, V\right)$ with weight 1 ;

- for each $x \in X_{2}$, an edge $(x, t)$ with weight $\Delta(P, x)$.

We refer the reader to Figure 2 for an illustration. (Once this graph is constructed, any standard function to compute a flow $\phi$ of maximal value can of course be used). We are now in a position to detail the main function 
CheckCowinner(.).

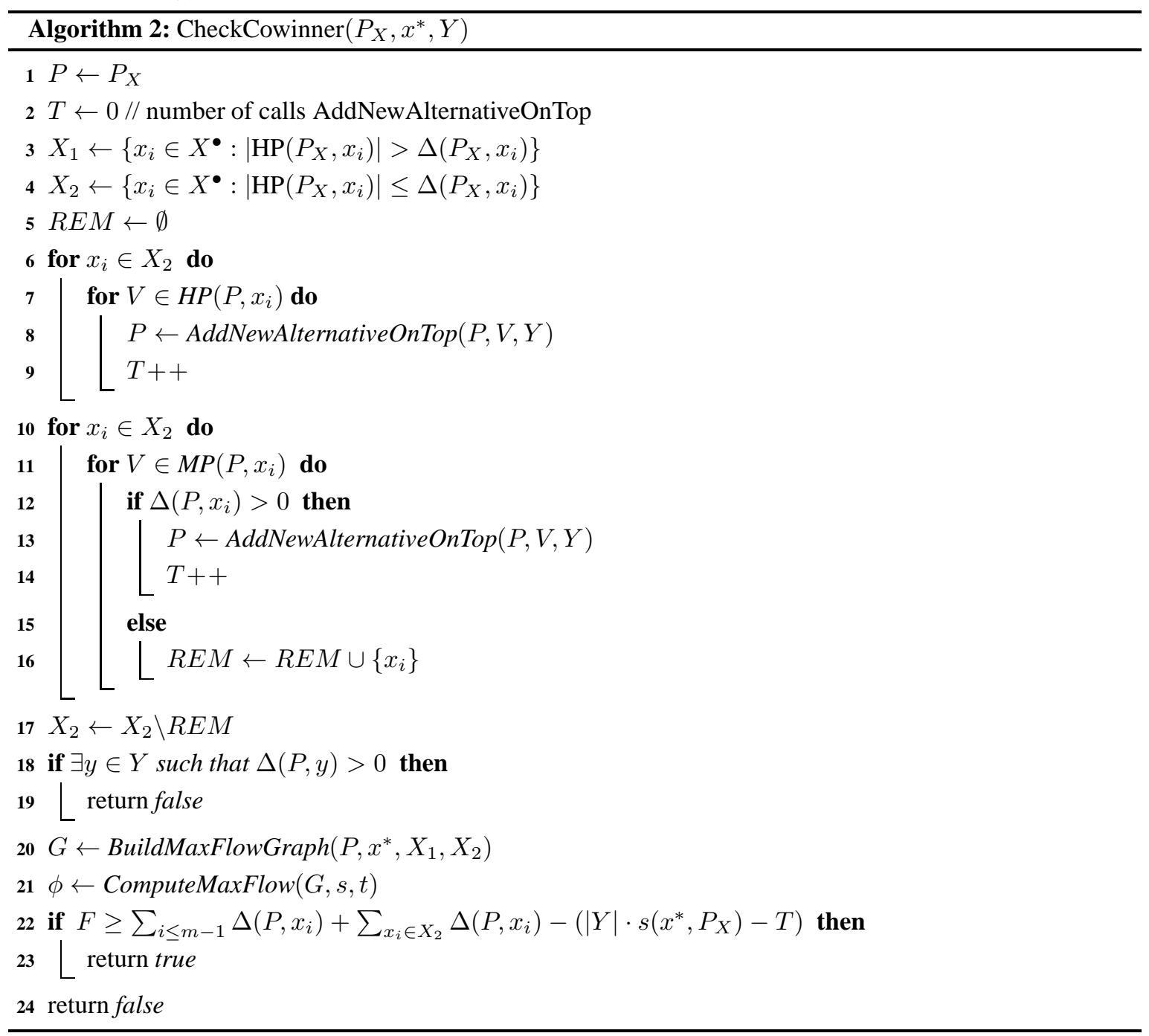

Proposition 4 Given a profile $P_{X}$ on $X$, a candidate $x^{*} \in X$ and a set of new candidates $Y$, a call to algorithm CheckCowinner $\left(P_{X}, x^{*}, Y\right)$ returns in polynomial time the answer true if and only if there exists an extension of $P_{X}$ in which $x^{*}$ is a cowinner.

Proof: Algorithm 2 starts by partitioning $X^{\bullet}$ into $X_{1}$ and $X_{2}$ : an alternative $x \in X^{\bullet}$ is in $X_{1}$ if $\left|\operatorname{HP}\left(P_{X}, x\right)\right|>$ $\Delta\left(P_{X}, x_{i}\right)$ and in $X_{2}$ if $\left|\operatorname{HP}\left(P_{X}, x\right)\right| \leq \Delta\left(P_{X}, x\right)$.

Let $x \in X_{2}$. Then by item (3) of Proposition 3 , for each vote in $V \in \operatorname{HP}(P, x)$, we can safely put one candidate from $Y$ in the top position of $V$; this is done in the first phase of Algorithm 2 lines 6 to 9 . Note that after adding a new candidate on top of a vote $V \in \operatorname{HP}(P, x)$ and after updating $P$, the modified vote will no longer belong to $\operatorname{HP}(P, x)$. Instead, it will now belong to $\operatorname{MP}\left(P, x^{\prime}\right)$ for some other candidate $x^{\prime}$.

When Phase 1 is over, the score of $x \in X_{2}$ may still need to be lowered down, which can be done next by using votes from $\operatorname{MP}\left(P_{X}, x\right)$. This is what Phase 2 does, from line 10 to line 16 There are three possibilities:

1. $\left|\operatorname{HP}\left(P_{X}, x\right)\right|=\Delta\left(P_{X}, x\right)$. In this case, the votes in $\operatorname{HP}\left(P_{X}, x\right)$ are sufficient to make $x^{*}$ catch up $x$ : 
after Phase 1, we have $\Delta\left(P_{X}, x\right)=0$ and Phase 2 is void; we are done with $x$.

2. $\left|\mathrm{HP}\left(P_{X}, x\right)\right|<\Delta\left(P_{X}, x\right)$ and $\left|\mathrm{HP}\left(P_{X}, x\right)\right|+\left|\operatorname{MP}\left(P_{X}, x\right)\right| \geq \Delta\left(P_{X}, x\right)$ : in this case, to make $x^{*}$ catch up $x$, it is enough to take $\Delta\left(P_{X}, x\right)-\left|\operatorname{HP}\left(P_{X}, x\right)\right|$ arbitrary votes in $\operatorname{MP}\left(P_{X}, x\right)$ and add one new candidate on top of them; this is what Phase 2 does, and after that we are done with $x$.

3. $\left|\operatorname{HP}\left(P_{X}, x\right)\right|+\left|\operatorname{MP}\left(P_{X}, x\right)\right|<\Delta\left(P_{X}, x\right)$ : in this case, because of Proposition 3, we know that it is safe to add one new candidate on top of all votes of $\operatorname{MP}\left(P_{X}, x\right)$; this is what Phase 2 does; after that, we still need to lower down the score of $x$, which will require to add new candidates on top of votes of $\mathrm{LP}\left(P_{X}, x\right)$.

If at this point a newly added candidate has a score higher than $x^{*}$, then $x^{*}$ cannot win, and we can stop the program (line 19.)

For readability, let us denote by $\widetilde{P}$ the profile obtained after Phases 1 and 2 . For each $x \in X_{2}$ satisfying condition 3 , the only way to reduce $\Delta(\widetilde{P}, x)$ is to put two candidates of $Y$ within the top two positions in a vote of $\operatorname{LP}(\widetilde{P}, x)$, because in Phases 1 and 2 we have used up all the votes in $\operatorname{HP}(P, x)$ and $\operatorname{MP}(P, x)$. Now, reducing $\Delta(\widetilde{P}, x)$ by one unit will cost us two candidates in $Y$, but meanwhile, $\Delta\left(\widetilde{P}, x^{\prime}\right)$ is also reduced by one unit, where $x^{\prime}$ is the candidate ranked in the second position in $V$. We must have $x^{\prime} \in X_{1}$. We note that $\bigcup_{x \in X_{2}} \operatorname{LP}\left(P_{X}, x\right) \subseteq \bigcup_{x^{\prime} \in X_{1}} \operatorname{HP}\left(P_{X}, x^{\prime}\right)$. Choosing optimally the votes in $\operatorname{LP}\left(P_{X}, x\right)$ for each $x \in X_{2}$ can be done by solving an integral max-flow instance which is build by algorithm BuildMaxFlowGraph (note that in case where either $X_{1}$ or $X_{2}$ is empty, we just assume that the flow has a null value).

Let us show that $x^{*}$ is a possible cowinner if and only if the value of the flow from $s$ to $t$ is at least $\sum_{i \leq m-1} \Delta\left(\widetilde{P}, x_{i}\right)+\sum_{x_{i} \in X_{2}} \Delta\left(\widetilde{P}, x_{i}\right)-\left(|Y| \cdot s\left(x^{*}, P_{X}\right)-T\right)$. Observe that the flow does not necessarily bring all $\Delta\left(\widetilde{P}, x_{i}\right)$ to 0 , therefore we sometimes need a postprocessing consisting of adding further new candidates on top of some votes (see steps 2 and 3 below).

Suppose first that the above max-flow instance has a solution whose value which is at least

$$
\sum_{i \leq m-1} \Delta\left(\widetilde{P}, x_{i}\right)+\sum_{x_{i} \in X_{2}} \Delta\left(\widetilde{P}, x_{i}\right)-\left(|Y| \cdot s\left(x^{*}, P_{X}\right)-T\right)
$$

We show how to solve our cowinner problem from the solution to this flow problem. Because the instance is integral, there must exist an integral solution. We arbitrarily choose one integral solution $\phi$ (as returned by ComputeMaxFlox), which assigns to each edge $\left(x_{i}, x_{j}\right)$ an integer $\phi\left(x_{i}, x_{j}\right)$ which represents the value of the flow on this edge. Here, we give a procedure which produces an extension $P$ of $P_{X}$ where $x^{*}$ is a cowinner:

1. For each $x_{i} \in X_{2}$ and each $V \in \operatorname{LP}\left(\widetilde{P}, x_{i}\right)$, if there is a flow from $x_{i}$ to $x_{j}$ via $V$, then we obtain $V^{*}$ from $V$ by putting two candidates from $Y$ in the top positions (that is, both $\Delta\left(\widetilde{P}, x_{i}\right)$ and $\Delta\left(\widetilde{P}, x_{j}\right)$ are reduced by 1 , which comes at the cost of using candidates in $Y$ twice). It is possible since $|Y| \geq 2$.

2. For each $x_{i} \in X_{2}$, if $\phi\left(x_{i}, t\right)<\Delta\left(\widetilde{P}, x_{i}\right)$, then we arbitrarily choose $\Delta\left(\widetilde{P}, x_{i}\right)-\phi\left(x_{i}, t\right)$ votes $V \in$ $\operatorname{LP}\left(\widetilde{P}, x_{i}\right)$ among those which haven't been selected in the previous step, and obtain $V^{*}$ by putting two candidates from $Y$ in the top two positions (again, we will specify how to choose the two candidates from $Y$ later). It is possible since $|Y| \geq 2$.

3. For each $x_{j} \in X_{1}$, if $\phi\left(s, x_{j}\right)<\Delta\left(\widetilde{P}, x_{i}\right)$, then we arbitrarily choose $\Delta\left(\widetilde{P}, x_{i}\right)-\phi\left(s, x_{j}\right)$ votes $V \in \operatorname{HP}\left(\widetilde{P}, x_{j}\right)$ such that $V^{*}$ is not defined above (in (1) or (2)), and then we obtain $V^{*}$ by putting 
exactly one candidate from $Y$ in the top position of $V$. This is possible because, by construction, $\left|\operatorname{HP}\left(\widetilde{P}, x_{j}\right)\right|=\left|\operatorname{HP}\left(P, x_{j}\right)\right| \geq \Delta\left(P, x_{i}\right) \geq \Delta\left(\widetilde{P}, x_{i}\right)$ for $x_{j} \in X_{1}$.

4. For each $V^{*}$, if a candidate $y \in Y$ is not selected for one of the first two positions, then it is ranked at the bottom position.

In the above procedure (similarly to what is done in Algorithm 1), priority is given to candidates from $Y$ with the lowest score (or the lowest index, in case of ties) when it comes to choose those to be added on top of the votes.

Let us now determine the number of times that new candidates from $Y$ are inserted on top of the votes. Recall that until line 20 of the algorithm, we have used the candidates from $Y$ exactly $T$ times. Now consider the four-step procedure described above. Observe that to reduce by one unit the score deficit with respect to one candidate, steps 1 and 3 require one occurrence of a candidate of $Y$ (step 1 uses two occurrences but reduces the score deficit with respect to two candidates), while step 2 requires two occurrences. Thus, for each $i \leq m-1$, we have to use $\Delta\left(\widetilde{P}, x_{i}\right)$ times the candidates from $Y$, plus the additional occurrences required in step 2. More precisely, step 2 requires, for each $x_{i} \in X_{2}, \Delta\left(\widetilde{P}, x_{i}\right)-\phi\left(x_{i}, t\right)$ additional occurrences of new candidates in the completed votes. Therefore, the total number of times that the candidates of $Y$ are ranked either in first or second position (denoted $s_{Y}$ for readability), is such that:

$$
\begin{aligned}
s_{Y} & \leq \sum_{i \leq m-1} \Delta\left(\widetilde{P}, x_{i}\right)+\left(\sum_{x_{i} \in X_{2}} \Delta\left(\widetilde{P}, x_{i}\right)-\sum_{x_{i} \in X_{2}} \phi\left(x_{i}, t\right)\right) \\
& =\sum_{i \leq m-1} \Delta\left(\widetilde{P}, x_{i}\right)+\left(\sum_{x_{i} \in X_{2}} \Delta\left(\widetilde{P}, x_{i}\right)-\phi\right)
\end{aligned}
$$

But we also have :

$$
\phi \geq \sum_{i \leq m-1} \Delta\left(\widetilde{P}, x_{i}\right)+\sum_{x_{i} \in X_{2}} \Delta\left(\widetilde{P}, x_{i}\right)-\left(|Y| \cdot s\left(x^{*}, P_{X}\right)-T\right)
$$

By combining (2) and (3), we thus get :

$$
\begin{aligned}
s_{Y} & \leq|Y| \cdot s\left(x^{*}, P_{X}\right)-T \\
& \leq|Y| \cdot s\left(x^{*}, P_{X}\right)
\end{aligned}
$$

That is, our algorithm will put candidates from $Y$ in the top two positions in the extension no more than $|Y| \cdot s\left(x^{*}, P_{X}\right)$ times. Because the addition of new candidates is done in a cyclic order, each new candidate will eventually appear at most $s\left(x^{*}, P_{X}\right)$ in the top two positions of the votes. Thus, the score of these new candidates will not exceed that of $x^{*}$. It follows that $x^{*}$ is a cowinner in $P^{*}$, since for all other candidates $x_{i} \in X$, we have $\Delta\left(P^{*}, x_{i}\right) \leq 0$.

Next, we show that if $x^{*}$ is a possible cowinner, then the value of a max-flow must be at least

$$
\sum_{i \leq m-1} \Delta\left(\widetilde{P}, x_{i}\right)+\sum_{x_{i} \in X_{2}} \Delta\left(\widetilde{P}, x_{i}\right)-\left(|Y| \cdot s\left(x^{*}, P_{X}\right)-T\right)
$$

Due to Proposition 3, each extension profile $P^{*}$ of $P_{X}$ where $x^{*}$ becomes a cowinner to the problem instance can be converted to a profile $\widetilde{P}$ as in the steps before line 20 in the algorithm. Now, for each $x_{i} \in X_{2}$, let $l_{i}$ denote the number of votes $V \in \operatorname{LP}\left(\widetilde{P}, x_{i}\right)$ such that in its extension $V^{*}$, the top two positions are the 
candidates of $Y$. We must have that $l_{i} \geq \Delta\left(\widetilde{P}, x_{i}\right)$. For every $x_{i} \in X_{2}$, we arbitrarily choose $l_{i}-\Delta\left(\widetilde{P}, x_{i}\right)$ such votes, and move the first ranked candidate to the bottom position. For each $x_{j} \in X_{1}$, let $l_{j}$ denote the number of votes $V \in \operatorname{HP}\left(\widetilde{P}, x_{j}\right) \cup \operatorname{MP}\left(\widetilde{P}, x_{j}\right)$ such that in its extension $V^{*}$, a candidate from $Y$ is ranked in the top position. We must have that $l_{j} \geq \Delta\left(\widetilde{P}, x_{j}\right)$. For every $x_{j} \in X_{1}$, we arbitrarily choose $l_{j}-\Delta\left(\widetilde{P}, x_{j}\right)$ such votes, and move the first ranked candidate to the bottom position.

Now, let there be a flow from $x_{j} \in X_{1}$ to $x_{i} \in X_{2}$ via $V$ if $V \in \operatorname{LP}\left(\widetilde{P}, x_{i}\right)$ and the top two positions in $V^{*}$ are both in $Y$. This defines a flow whose value is at least $\sum_{x_{i} \in X_{2}} \Delta\left(\widetilde{P}, x_{i}\right)-\sum_{x_{j} \in X_{1}}\left(l_{j}-\Delta\left(\widetilde{P}, x_{j}\right)\right)$. Because the score of each candidate of $Y$ is no more than $s\left(x^{*}, P_{X}\right)$, we know that $|Y| \cdot s\left(x^{*}, P_{X}\right)-T \geq \sum_{i \leq m-1} l_{i}$. Actually, $|Y| \cdot s\left(x^{*}, P_{X}\right)$ is the maximum score that the whole set of new candidates of $Y$ can reach in such a way that $x^{*}$ is a cowinner. In the partial profile $\widetilde{P}$ (line 20 of Algorithm CheckCowinner $\left(P_{X}, x^{*}, Y\right)$ ), the global score of $Y$ is $T$. Finally, since $\sum_{i \leq m-1} l_{i}+T$ corresponds to the global score that $Y$ has in profile $P^{*}$ (where $x^{*}$ becomes a cowinner), we get $|Y| \cdot s\left(x^{*}, P_{X}\right) \geq \sum_{i \leq m-1} l_{i}+T$.

Hence, $|Y| \cdot s\left(x^{*}, P_{X}\right)-T \geq \sum_{i \leq m-1} l_{i} \geq \sum_{x_{i} \in X_{2}} \Delta\left(\widetilde{P}, x_{i}\right)+\sum_{x_{j} \in X_{1}} l_{j}$, or equivalently, $-\sum_{x_{j} \in X_{1}} l_{j} \geq$ $\sum_{x_{i} \in X_{2}} \Delta\left(\widetilde{P}, x_{i}\right)-\left(|Y| \cdot s\left(x^{*}, P_{X}\right)-T\right)$. Hence, we get:

$$
\begin{aligned}
\phi & \geq \sum_{x_{i} \in X_{2}} \Delta\left(\widetilde{P}, x_{i}\right)-\sum_{x_{j} \in X_{1}}\left(l_{j}-\Delta\left(\widetilde{P}, x_{j}\right)\right) \\
& =\sum_{i \leq m-1} \Delta\left(\widetilde{P}, x_{i}\right)-\sum_{x_{j} \in X_{1}} l_{j} \\
& \geq \sum_{i \leq m-1} \Delta\left(\widetilde{P}, x_{i}\right)+\sum_{x_{i} \in X_{2}} \Delta\left(\widetilde{P}, x_{i}\right)-\left(|Y| s\left(x^{*}, P_{X}\right)-T\right)
\end{aligned}
$$

Thus, we have shown that $x^{*}$ is a possible cowinner if and only if the value of the flow from $s$ to $t$ is at least $\sum_{i \leq m-1} \Delta\left(\widetilde{P}, x_{i}\right)+\sum_{x_{i} \in X_{2}} \Delta\left(\widetilde{P}, x_{i}\right)-\left(|Y| \cdot s\left(x^{*}, P_{X}\right)-T\right)$. This concludes the proof.

Corollary 1 Deciding whether $x^{*}$ is a possible cowinner for 2-approval with respect to the addition of new candidates is in $\mathrm{P}$.

To better understand Algorithm 1, we will now run it step by step on the example introduced previously.

Example 2 Consider the profile described in Example 1. We assume the number of new candidates is $k=3$. First, the initial scores of the candidates are $s\left(x^{*}, P_{X}\right)=4, s\left(x_{1}, P_{X}\right)=7, s\left(x_{2}, P_{X}\right)=7, s\left(x_{3}, P_{X}\right)=8$, $s\left(x_{4}, P_{X}\right)=6$ and $s\left(x_{5}, P_{X}\right)=4$ and $s\left(x_{6}, P_{X}\right)=2$. The candidates whose score exceeds that of $x^{*}$ are $x_{1}$, $x_{2}, x_{3}$ and $x_{4}$, with the score differences $\Delta\left(P, x_{1}\right)=3, \Delta\left(P, x_{2}\right)=3, \Delta\left(P, x_{3}\right)=4$ and $\Delta\left(P, x_{4}\right)=2$. At first phase, we check if there are candidates $x_{i}$ for which $\left|H P\left(P_{X}, x_{i}\right)\right| \leq \Delta\left(P_{X}, x_{i}\right)$. This is the case for $x_{1}$, $x_{2}$ and $x_{3}$, thus we put one new candidate on top of $v_{8}, v_{9}, v_{13}$ and $v_{14}$. The updated table is as follows:

\begin{tabular}{|l|l|l|l|l|}
\hline & HP & MP & LP & $\Delta\left(P, x_{i}\right)$ \\
\hline$x_{1}$ & & $v_{1}$ & $v_{5}, v_{6}, v_{7}$ & 1 \\
$x_{2}$ & & $v_{8}^{\prime}, v_{9}^{\prime}$ & $v_{10}, v_{11}, v_{12}$ & 2 \\
$x_{3}$ & & $v_{13}^{\prime}, v_{14}^{\prime}$ & $v_{15}, v_{16}, v_{17}, v_{18}$ & 3 \\
$x_{4}$ & $v_{5}, v_{6}, v_{10}, v_{15}, v_{16}$ & & $v_{19}$ & 2 \\
\hline
\end{tabular}

Here, $v_{i}^{\prime}$ refers to the vote $v_{i}$ to which new candidates have been added. 
At the second phase, $X_{2}=\left\{x_{1}, x_{2}, x_{3}\right\}$ and $X_{1}=\left\{x_{4}\right\}$ (we do not worry about $x_{5}$ and $x_{6}$ for which nothing special has to be done). We put one new candidate on top of $v_{1}, v_{8}^{\prime}, v_{9}^{\prime}, v_{13}^{\prime}$ and $v_{14}^{\prime}$, and we are done with $x_{1}$ and $x_{2}\left(\right.$ since $\Delta\left(P, x_{1}\right)=0$ and $\left.\Delta\left(P, x_{2}\right)=0\right)$. The profile is now $\widetilde{P}$ and the updated table is :

\begin{tabular}{|l|l|l|l|l|}
\hline & HP & MP & LP & $\Delta\left(\widetilde{P}, x_{i}\right)$ \\
\hline$x_{3}$ & & & $v_{15}, v_{16}, v_{17}, v_{18}$ & 1 \\
$x_{4}$ & $v_{5}, v_{6}, v_{10}, v_{15}, v_{16}$ & & $v_{19}$ & 2 \\
\hline
\end{tabular}

So far we have used the new candidates 9 times, and $s\left(x^{*}, \widetilde{P}\right)=4$, therefore if we have less than three new candidates we stop ( $x^{*}$ is not a possible cowinner) otherwise we continue. Now the situation is as follows and we have to solve the corresponding maxflow problem (we omit the value of edges when it equals 1).

\begin{tabular}{|c|c|c|c|c|c|c|c|c|c|c|c|c|c|c|c|c|c|c|}
\hline$v_{1}$ & $v_{2}$ & $v_{3}$ & $v_{4}$ & $v_{5}$ & $v_{6}$ & $v_{7}$ & $v_{8}$ & $v_{9}$ & $v_{10}$ & $v_{11}$ & $v_{12}$ & $v_{13}$ & $v_{14}$ & $v_{15}$ & $v_{16}$ & $v_{17}$ & $v_{18}$ & $v_{19}$ \\
\hline$\bullet$ & $x_{1}$ & $x_{2}$ & $x_{3}$ & $x_{1}$ & $x_{1}$ & $x_{1}$ & $\bullet$ & $\bullet$ & $x_{2}$ & $x_{2}$ & $x_{2}$ & $\bullet$ & $\bullet$ & $x_{3}$ & $x_{3}$ & $x_{3}$ & $x_{3}$ & $x_{4}$ \\
\hline$x^{*}$ & $x^{*}$ & $x^{*}$ & $x^{*}$ & $x_{4}$ & $x_{4}$ & $x_{5}$ & $\bullet$ & $\bullet$ & $x_{4}$ & $x_{5}$ & $x_{5}$ & $\bullet$ & $\bullet$ & $x_{4}$ & $x_{4}$ & $x_{5}$ & $x_{6}$ & $x_{6}$ \\
\hline$\vdots$ & : & $\vdots$ & : & : & : & : & : & : & : & : & : & $\vdots$ & : & : & : & : & : & $:$ \\
\hline
\end{tabular}

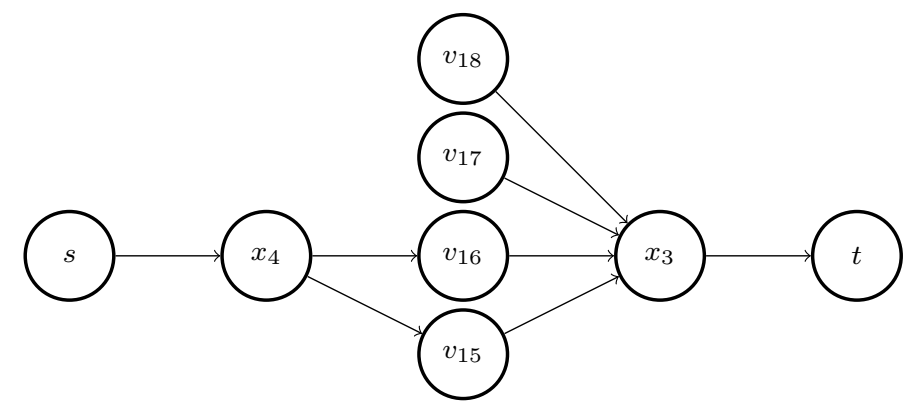

Figure 2: The flow graph returned by BuildMaxFlowGraph $\left(\widetilde{P}, x^{*},\left\{x_{4}\right\},\left\{x_{3}\right\}\right)$.

The maximum flow has value 1 and is obtained for instance by having a flow 1 for instance through the edges $s \rightarrow x_{4}, x_{4} \rightarrow v_{16}, v_{16} \rightarrow x_{3}, x_{3} \rightarrow t$ (going through $v_{15}$ is an equally good option). Therefore we place two new candidates on top of $v_{16}$, which has the effect of making the score of $x_{3}$ and $x_{4}$ decrease by one unit each. We still have to make the score of $x_{4}$ decrease by one unit, and for this we must place one new candidates on top of any of the votes $v_{5}, v_{6}, v_{10}, v_{15}$ (say $\left.v_{5}\right)$. In total we will have used the new candidates 12 times, therefore, $c$ is a possible cowinner if and only if the number of new candidates is at least 3. A possible extension (with 3 new candidates) is as follows:

\begin{tabular}{|c|c|c|c|c|c|c|c|c|c|c|c|c|c|c|c|c|c|c|}
\hline$v_{1}$ & $v_{2}$ & $v_{3}$ & $v_{4}$ & $v_{5}$ & $v_{6}$ & $v_{7}$ & $v_{8}$ & $v_{9}$ & $v_{10}$ & $v_{11}$ & $v_{12}$ & $v_{13}$ & $v_{14}$ & $v_{15}$ & $v_{16}$ & $v_{17}$ & $v_{18}$ & $v_{19}$ \\
\hline $\mathrm{y}_{1}$ & $x_{1}$ & $x_{2}$ & $x_{3}$ & $\mathrm{y}_{2}$ & $x_{1}$ & $x_{1}$ & $\mathbf{y}_{1}$ & $\mathrm{y}_{2}$ & $x_{2}$ & $x_{2}$ & $x_{2}$ & $\mathbf{y}_{1}$ & $\mathrm{y}_{2}$ & $x_{3}$ & $\mathbf{y}_{1}$ & $x_{3}$ & $x_{3}$ & $x_{4}$ \\
\hline$x^{*}$ & $x^{*}$ & $x^{*}$ & $x^{*}$ & $x_{1}$ & $x_{4}$ & $x_{5}$ & $\mathbf{y}_{3}$ & уз & $x_{4}$ & $x_{5}$ & $x_{5}$ & уз & $\mathbf{y}_{3}$ & $x_{4}$ & $\mathbf{y}_{2}$ & $x_{5}$ & $x_{6}$ & $x_{6}$ \\
\hline
\end{tabular}

\section{4 $K$-approval, two new candidates}

Let $X=\left\{x^{*}\right\} \cup\left\{x_{1}, \ldots, x_{p}\right\}$ be the set of (initial) candidates, $x^{*}$ being the candidate that we want to make a cowinner, $Y=\left\{y_{1}, y_{2}\right\}$ the two new candidates, and $P_{X}=\left\langle V_{1}, \ldots, V_{n}\right\rangle$ the initial profile, where each $V_{i}$ is a sequence of $K$ candidates in $X$. We first introduce the following notation: 
- For each $x \in X, U^{P_{X}}(x)$ is the number of votes in $P_{X}$ whose candidates ranked $K-1$ and $K$ are respectively $x$ and $x^{*}$, and $T^{P_{X}}(x)=S_{K-2}\left(x, P_{X}\right)+U^{P_{X}}(x)$. (Recall that $S_{K-2}\left(x, P_{X}\right)$ is the number of voters in $P_{X}$ who rank $x$ in the first $K-2$ positions.)

We establish the following lemma.

Lemma 1 For each $x \in X$, there exists a completion $Q$ of $P_{X}$ by adding two candidates such that $S_{K}(x, Q) \leq$ $S_{K}\left(x^{*}, Q\right)$ if and only if $T^{P_{X}}(x) \leq S_{K}\left(x^{*}, P_{X}\right)$.

Proof: Assume $T^{P_{X}}(x)>S_{K}\left(x^{*}, P_{X}\right)$, and let $Q$ be a completion of $P_{X}$ by adding two candidates in which $x^{*}$ is a cowinner. Let us partition $P_{X}$ into $P_{1}, P_{2}$ and $P_{3}$, as follows: every vote in $P_{1}$ is such that the candidates ranked $K-1$ and $K$ are respectively $x$ and $x^{*} ; P_{2}$ contains all votes ranking $x$ in the first $K-2$ positions; and $P_{3}$ contains all other votes in $P_{X}$. Let $Q_{1}, Q_{2}$ and $Q_{3}$ be the corresponding votes in $Q$, and let $\alpha$ be the number of votes in $Q_{1}$ where the two new candidates have been placed in the first $K$ positions, thus eliminating both $x$ and $x^{*}$ from the $K$ first positions; clearly, we have $S_{K}\left(x, Q_{1}\right)=S_{K}\left(x, P_{1}\right)-\alpha=U^{P_{X}}(x)-\alpha$ and $S_{K}\left(x^{*}, Q_{1}\right) \leq S_{K}\left(x^{*}, P_{1}\right)-\alpha$ (the inequality can be strict, in case there are some votes in $Q_{1}$ where only one new candidate was placed in the first $K$ positions). Now, regardless of the position of the two new candidates, we have $S_{K}\left(x, Q_{2}\right)=S_{K-2}\left(x, P_{2}\right)$. We get $S_{K}(x, Q)=S_{K}\left(x, Q_{1}\right)+S_{K}\left(x, Q_{2}\right)+S_{K}\left(x, Q_{3}\right) \geq$ $U^{P_{X}}(x)-\alpha+S_{K-2}\left(x, P_{2}\right)=T^{P_{X}}(x)-\alpha$, whereas $S_{K}\left(x^{*}, Q\right) \leq S_{K}\left(x^{*}, P_{X}\right)-\alpha$. The initial assumption $T^{P_{X}}(x)>S_{K}\left(x^{*}, P_{X}\right)$ implies $T^{P_{X}}(x)-\alpha>S_{K}\left(x^{*}, P_{X}\right)-\alpha$, therefore $S_{K}(x, Q)>S_{K}\left(x^{*}, Q\right)$.

Conversely, assume $T^{P_{X}}(x) \leq S_{K}\left(x^{*}, P_{X}\right)$, and let us build $Q$ as follows: we introduce one new candidate on top of each vote of $P_{X}$ that ranks $x$ in position $K$, and two new candidates on top of each vote of $P_{X}$ that ranks $x$ in position $K-1$ and $x^{\prime} \neq x^{*}$ in position $K$. It is easy to check that $S_{K}\left(x^{*}, Q\right)=S_{K}\left(x^{*}, P_{X}\right)$. Now, the only votes of $Q$ where $x$ remains among the first $K$ position are those of $Q_{1}$ and of $Q_{2}$, therefore $S_{K}(x, Q)=T^{P_{X}}(x) \leq S_{K}\left(x^{*}, P_{X}\right)=S_{K}\left(x^{*}, Q\right)$.

Proposition 5 Deciding whether $x^{*}$ is a possible cowinner for $K$-approval with respect to the addition of 2 new candidates is in $\mathrm{P}$.

Proof: A consequence of Lemma 1 is that if $T^{P_{X}}(x)>S_{K}\left(x^{*}, P_{X}\right)$ for some $x$, then $x^{*}$ cannot be a possible cowinner in $P_{X}$ under 2-approval with 2 new candidates; and obviously, checking whether $T^{P_{X}}(x)>$ $S_{K}\left(x^{*}, P_{X}\right)$ holds for some $x$ can be done in polynomial time. Therefore, from now on, we assume that $T^{P_{X}}(x) \leq S_{K}\left(x^{*}, P_{X}\right)$ holds for every $x \in X$ - assuming this will not change the complexity of the problem.

We now give a polynomial reduction from the possible cowinner problem for $K$-approval and 2 new candidates to the possible cowinner problem for 2-approval and 2 new candidates, which we already know to be polynomial. Let $\left\langle N, X, P_{X}, 2\right\rangle$ be an instance of the possible cowinner problem for $K$-approval with respect to the addition of 2 new candidates. We build an instance $\left\langle N^{\prime}, X^{\prime}, R_{X^{\prime}}, 2\right\rangle$ of the possible cowinner problem for 2 -approval with respect to the addition of 2 new candidates in the following way. The profile $P_{X}$ is translated into the following profile $R=R_{X^{\prime}}$ :

- the set of candidates is $X^{\prime}=X \cup\left\{z_{j}, 1 \leq j \leq \sum_{x \in X \backslash\left\{x^{*}\right\}} S_{K-2}\left(x, P_{X}\right)\right\} \cup\left\{z_{j}^{\prime}, 1 \leq j \leq S_{K-2}\left(x^{*}, P_{X}\right)\right\}$, where all $z_{j}$ and $z_{j}^{\prime}$ are fresh candidates; 
- for every vote $V_{i}$ in $P_{X}$, we have in $R$ a vote $W_{i}$ including the candidates ranked in positions $K-1$ and $K$ of $V_{i}$, and then the remaining candidates in any order. We denote by $R_{1}$ be the resulting set of votes;

- for every $x \in X \backslash\left\{x^{*}\right\}$, we have $S_{K-2}\left(x, P_{X}\right)$ votes $x z_{j}$, and then the remaining candidates in any order. We denote by $R_{2}$ the resulting set of votes;

- similarly, we have $S_{K-2}\left(x^{*}, P_{X}\right)$ votes $z_{j}^{\prime} x^{*}$, and then the remaining candidates in any order. We denote by $R_{3}$ the resulting set of votes.

We note that if $x \in X$ then $S_{K}\left(x, P_{X}\right)=S_{2}(x, R)$, and for every fresh candidate $z, S_{2}(z, R)=1$. Without loss of generality we assume $S_{K}\left(x^{*}, P_{X}\right) \geq 1$ (otherwise we know for sure that $x^{*}$ cannot be a possible cowinner).

We decompose the rest of the proof into two lemmas.

Lemma 2 If $x^{*}$ is a possible cowinner for $K$-approval with 2 new candidates in $P_{X}$, then it is is a possible cowinner for 2-approval with 2 new candidates in $R$.

Proof: Suppose that $x^{*}$ is a possible cowinner for $K$-approval with 2 new candidates $Y=\left\{y_{1}, y_{2}\right\}$ in $P_{X}$ and let $P^{\prime}=\left\langle V_{1}^{\prime}, \ldots, V_{n}^{\prime}\right\rangle$ be an extension of $P_{X}$ with two new candidates where $x^{*}$ is a cowinner. Let us use these two new candidates in the same way in $R$ : every time a new candidate is used for being placed on top of $V_{i}$, it is also used for being placed on top of $W_{i}$. Let $R^{\prime}$ be the resulting profile. All candidates in $X$ have the same scores in $P_{X}$ and in $R$, they also will have the same scores in $P^{\prime}$ and $R^{\prime}$; as for the fresh candidates $z_{j}, z_{j}^{\prime}, S_{2}\left(z_{j}, R^{\prime}\right)=S_{2}\left(z_{j}^{\prime}, R^{\prime}\right)=1 \leq S_{2}\left(x^{*}, R^{\prime}\right)$; therefore, $x^{*}$ is a cowinner in $R^{\prime}$ and a possible cowinner for 2-approval with 2 new candidates in $R$.

Lemma 3 If $x^{*}$ is a possible cowinner for $K$-approval with 2 new candidates in $R$, then it is a possible cowinner for 2-approval in $P_{X}$.

Proof: Suppose that $x^{*}$ is a possible cowinner for 2 -approval with 2 new candidates $Y=\left\{y_{1}, y_{2}\right\}$ in $R$, and let $R^{\prime}$ be a completion of $R$ where $x^{*}$ is a cowinner for 2 -approval. Let us write $R^{\prime}=R_{1}^{\prime} \cup R_{2}^{\prime} \cup R_{3}^{\prime}$, where $R_{1}^{\prime}$ (resp. $R_{2}^{\prime}, R_{3}^{\prime}$ ) consists in the completions of the votes in $R_{1}$ (resp. $R_{2}, R_{3}$ ). By a slight abuse of language we denote by $R_{1}, R_{1}^{\prime}$ etc. only the part of the votes in $R_{1}, R_{1}^{\prime}$ etc. consisting of the top two candidates only.

We first claim that we can assume without loss of generality that $R_{2}^{\prime}=R_{2}$ and $R_{3}^{\prime}=R_{3}$ that is, the only votes in $R^{\prime}$ where some new candidates have been placed on one of the top two positions are in $R_{1}^{\prime}$. Suppose this is not the case; then we are in one of the following four situations: (1) there is a vote in $R_{2}^{\prime}$ of the form $y_{j} x_{i}$, where $y_{j} \in Y$ and $x_{i} \in X$, or (2) there is a vote in $R_{2}^{\prime}$ of the form $y_{1} y_{2}$ or $y_{2} y_{1}$, or (3) there is a vote in $R_{3}^{\prime}$ of the form $y_{i} z_{j}^{\prime}$ or (4) there is a vote in $R_{3}^{\prime}$ of the form $y_{1} y_{2}$ or $y_{2} y_{1}$. Consider first cases (1), (3) and (4). Take one of these votes in $R_{2}^{\prime}$ (case (1)) or in $R_{3}^{\prime}$ (cases (3) or (4)) and replace it by the original vote $x z_{j}$ in $R_{2}$ (case 1 ) or in $z_{j}^{\prime} x^{*}$ in $R_{3}^{\prime}$ (cases (3) or (4)). Let $R^{\prime \prime}$ be the profile obtained. We have $S_{2}\left(x^{*}, R^{\prime \prime}\right) \geq S_{2}\left(x^{*}, R^{\prime}\right) \geq 1$, for every $x_{i} \in X, S_{2}\left(x_{i}, R^{\prime \prime}\right)=S_{2}\left(x_{i}, R^{\prime}\right)$, for every $z_{j}, S_{2}\left(z_{j}, R^{\prime \prime}\right) \leq 1$, and for every $z_{j}^{\prime}, S_{2}\left(z_{j}^{\prime}, R^{\prime \prime}\right) \leq 1$.

Therefore, when transforming $R^{\prime}$ into $R^{\prime \prime}$, the score of $x^{*}$ does not decrease whereas the score of all other candidates does not increase; because $x^{*}$ is a cowinner in $R^{\prime}$, it is still a cowinner in $R^{\prime \prime}$. Lastly, $R^{\prime \prime}$ is also an extension of $R$. 
By induction, if we perform this operation for each occurrence of cases (1), (3) or (4), we end up with a profile $R^{\prime \prime}$, which is an extension of $R$ for which situations (1), (3) and (4) do not occur, and such that $x^{*}$ is a cowinner for 2-approval in $R^{\prime \prime}$. Let $R^{\prime \prime}=R_{1}^{\prime \prime} \cup R_{2}^{\prime \prime} \cup R_{3}^{\prime \prime}=R_{1}^{\prime \prime} \cup R_{2}^{\prime \prime} \cup R_{3}$.

Now, consider case (2). Let $x_{i} z_{j}$ be one of the votes in $R_{2}$ corresponding to a vote $y_{1} y_{2}$ (or $y_{2} y_{1}$ ) in $R_{2}^{\prime \prime}$. Apply the following procedure in this order:

1. Assume that $x_{i}$ does not appear in $R_{1}^{\prime \prime}$ except in votes of the form $x_{i} x^{*}$, and let $R^{\prime \prime \prime}$ be the profile obtained from $R^{\prime \prime}$ by replacing the vote $y_{1} y_{2}$ in $R_{2}^{\prime \prime}$ by the original vote $x_{i} z_{j}$ in $R_{2}$. Then $S_{2}\left(x_{i}, R^{\prime \prime \prime}\right)=$ $S_{2}\left(x_{i}, R_{1}^{\prime \prime \prime}\right)+S_{2}\left(x_{i}, R_{2}^{\prime \prime \prime}\right) \leq S_{2}\left(x_{i}, R_{1}^{\prime \prime \prime}\right)+S_{2}\left(x_{i}, R_{2}\right)$. Now, $S_{2}\left(x_{i}, R^{\prime \prime \prime}\right) \leq S_{2}\left(x_{i}, R^{\prime \prime \prime}\right)=U^{P_{X}}\left(x_{i}\right)$ and $S_{2}\left(x_{i}, R_{2}\right)=S_{K-2}\left(x_{i}, P_{X}\right)$, therefore $S_{2}\left(x_{i}, R^{\prime \prime \prime}\right) \leq U^{P_{X}}\left(x_{i}\right)+S_{K-2}\left(x_{i}, P_{X}\right)=T^{P_{X}}\left(x_{i}\right) \leq$ $S_{K}\left(x^{*}, P_{X}\right)=S_{2}\left(x^{*}, R\right)=S_{2}\left(x^{*}, R^{\prime \prime \prime}\right)$. Therefore, $x^{*}$ is also a cowinner in $R^{\prime \prime \prime}$.

2. Now, assume that $x_{i}$ appears in at least one vote of $R_{1}^{\prime \prime}$ of the form $x^{*} x_{i}, x_{i} x_{j}$ or $x_{j} x_{i}$. If this is a vote of the form $x_{i} x_{j}$, we replace $y_{1} y_{2}$ in $R_{2}^{\prime \prime}$ by the original vote $x_{i} z_{j}$ in $R_{2}$ and the vote $x_{i} x_{j}$ by a vote $y_{1} y_{2}$. If this is a vote of the form $x^{*} x_{i}$ or $x_{j} x_{i}$, we replace $y_{1} y_{2}$ in $R_{2}^{\prime \prime}$ by the original vote $x_{i} z_{j}$ in $R_{2}$ and the vote $x^{*} x_{i}$ (resp. $x_{j} x_{i}$ ) by $y_{1} x^{*}$ (resp. $y_{1} x_{j}$ ). In all three cases, the score of all candidates remain the same after the transformation, except the score of $y_{2}$ and $z_{j}$, which can only decrease, therefore $x^{*}$ is still a cowinner after the transformation.

We perform this procedure on $x_{i}$ iteratively until all the votes $y_{1} y_{2}$ (or $y_{2} y_{1}$ ) in $R_{2}^{\prime \prime \prime}$ have been replaced by the original votes $x_{i} z_{j}$ in $R_{2}$. After doing this sequentially on all candidates of $X$ such that case (2) occurs, we end up with a profile $R^{\prime \prime \prime \prime}$ of $P$ such that $R_{2}^{\prime \prime \prime \prime}=R_{2}$ and $R_{3}^{\prime \prime \prime \prime}=R_{3}$ and $x^{*}$ is a cowinner in $R^{\prime \prime \prime \prime}$. This proves the claim.

Now, let $R^{\prime}$ be a completion of $R$ where $x^{*}$ is a cowinner for 2 -approval, where $R_{2}^{\prime}=R_{2}$ and $R_{3}^{\prime}=R_{3}$. From $R^{\prime}$ we build the following extension $P^{\prime}$ of $P$ : for every vote $W_{i} \in R_{1}$,

- if $W_{i}^{\prime}$ is of the form $x^{*} x^{\prime}$ then $V_{i}^{\prime}=W_{i}^{\prime}$;

- if $W_{i}^{\prime}$ is of the form $y_{i} x$ then $V_{i}^{\prime}$ is obtained from $V_{i}$ by placing $y_{i}$ on top;

- if $W_{i}^{\prime}$ is of the form $y_{1} y_{2}$ (or $\left.y_{2} y_{1}\right)$ then $V_{i}^{\prime}$ is obtained from $V_{i}$ by placing $\left\{y_{1}, y_{2}\right\}$ on top.

The scores of all candidates are the same in $P^{\prime}$ and in $R^{\prime}$, therefore $x^{*}$ is a cowinner in $P^{\prime}$ if only if it is a cowinner in $R^{\prime}$. Therefore, it is a cowinner in $P^{\prime}$, which means that $x^{*}$ is a possible cowinner for 2-approval in $P$.

We can now end the proof of Proposition 5, from Lemmas 2 and 3 we conclude that deciding whether $x^{*}$ is a possible cowinner for $K$-approval with respect to the addition of two candidates can be polynomially reduced to a problem of a deciding whether $x^{*}$ is a possible cowinner for 2-approval, which we know is in $\mathrm{P}$. 


\subsection{3-approval, 3 new candidates}

We will now see that the problems addressed in previous subsections constitute the frontier of what can be solved in polynomial-time for $K$-approval rules. In the rest of this paper, the hardness proofs will use reductions from the 3-dimensional matching (3-DM) problem.

Definition 4 An instance of 3-DM consists of a subset $\mathcal{C}=\left\{e_{1}, \ldots, e_{m}\right\} \subseteq A \times B \times C$ of triples, where $A, B, C$ are 3 pairwise disjoint sets of size $n^{\prime}$ with $A=\left\{a_{1}, \ldots, a_{n^{\prime}}\right\}, B=\left\{b_{1}, \ldots, b_{n^{\prime}}\right\}$ and $C=$ $\left\{c_{1}, \ldots, c_{n^{\prime}}\right\}$. For $z \in A \cup B \cup C, d(z)$ denotes the number of occurrences of $z$ in $\mathcal{C}$, that is the number of triples of $\mathcal{C}$ which contain $z$. A matching is a subset $M \subseteq \mathcal{C}$ such that no two elements in $M$ agree on any coordinate. The 3-DM problem consists in answering this question: does there exist a perfect matching $M$ on $\mathcal{C}$, that is, a matching of size $n^{\prime}$ ?

The 3-DM problem is known to be NP-complete (problem [SP1] page 221 in [19]), even with the restriction where $\forall z \in A \cup B \cup C, d(z) \in\{2,3\}$ (that is, no element of $A \cup B \cup C$ occurs in more than 3 triples, and each element of $A \cup B \cup C$ appears in at least 2 triples).

Proposition 6 Deciding if $x^{*}$ is a possible cowinner for 3-approval with respect to the addition of 3 new candidates, is an NP-complete problem.

Proof: This problem is clearly in NP. The hardness proof is based on a reduction from 3-DM (see Definition (4).

Let $I=(\mathcal{C}, A \times B \times C)$ be an instance of 3-DM with $n^{\prime} \geq 3$ and $\forall z \in A \cup B \cup C, d(z) \in\{2,3\}$. From $I$, we build an instance of the PcWNC problem as follows. The set $X$ of candidates contains $x^{*}, X_{1}=$ $\left\{x_{i}^{\prime}, y_{i}^{\prime}, z_{i}^{\prime}: 1 \leq i \leq n^{\prime}\right\}$ where $x_{i}^{\prime}, y_{i}^{\prime}, z_{i}^{\prime}$ correspond to elements of $A \cup B \cup C$ and a set $X_{2}$ of dummy candidates. We now describe the votes informally; their formal definition will follow. The set $N$ of voters contains $N_{1}=\left\{v^{e}: e \in \mathcal{C}\right\}$ and a set $N_{2}$ of dummy voters. For each voter, we only indicate her first three candidates. Thus, the vote of $v^{e}$ is $\left(x_{i}^{\prime}, y_{j}^{\prime}, z_{k}^{\prime}\right)$ where $e=\left(a_{i}, b_{j}, c_{k}\right) \in \mathcal{C}$. The preference of dummy voters are such that :

(i) the scores of the candidates in $X$ satisfy $\forall x \in X_{1}, S_{3}\left(x, P_{X}\right)=n^{\prime}+1, S_{3}\left(x^{*}, P_{X}\right)=n^{\prime}$ and $\forall x \in X_{2}$, $S_{3}\left(x, P_{X}\right)=1$

(ii) the vote of any voter of $N_{2}$ contains at most one candidate from $\left\{x_{i}^{\prime}, y_{i}^{\prime}, z_{i}^{\prime}: 1 \leq i \leq n^{\prime}\right\}$ in the first three positions, and if it contains one, then it is in top position.

Formally, the instance $\left\langle N, X, P_{X}, 3, x^{*}\right\rangle$ of the possible cowinner problem for 3-approval and 3 new candidates is described as follows: the set of voters is $N=N_{1} \cup N_{2}$ where $N_{1}=\left\{v^{e}: e \in \mathcal{C}\right\}$ and $N_{2}=N_{A} \cup N_{B} \cup N_{C} \cup N_{x^{*}}$, the set of candidates is $X=X_{1} \cup X_{2}$. For the candidates in $X$, we have $x^{*}$ together with :

- $X_{1}=X^{\prime} \cup Y^{\prime} \cup Z^{\prime}$ where $X^{\prime}=\left\{x_{1}^{\prime}, \ldots, x_{n^{\prime}}^{\prime}\right\}, Y^{\prime}=\left\{y_{1}^{\prime}, \ldots, y_{n^{\prime}}^{\prime}\right\}$ and $Z^{\prime}=\left\{z_{1}^{\prime}, \ldots, z_{n^{\prime}}^{\prime}\right\}$.

- $X_{2}=\left\{x_{i}^{*}: 1 \leq i \leq 2 n^{\prime}\right\} \cup\left\{x_{i}^{j}: 1 \leq i \leq n^{\prime}, 1 \leq j \leq 2\left(n^{\prime}-d\left(x_{i}\right)+1\right)\right\} \cup\left\{y_{i}^{j}: 1 \leq i \leq n^{\prime}\right.$, $\left.1 \leq j \leq 2\left(n^{\prime}-d\left(y_{i}\right)+1\right)\right\} \cup\left\{z_{i}^{j}: 1 \leq i \leq n^{\prime}, 1 \leq j \leq 2\left(n^{\prime}-d\left(z_{i}\right)+1\right)\right\}$.

Note that $n^{\prime}-d\left(x_{i}\right)+1 \geq 1$ since $d(z) \leq 3 \leq n^{\prime}$. 
For each voter $v_{i} \in N$, we only indicate her first three candidates (in the order of preference). The set of all $X$-votes $\mathcal{P}_{X}$ of the voters in $N$ is as follows :

- $N_{A}=\left\{v_{i, j}^{A}: 1 \leq i \leq n^{\prime}, 0 \leq j \leq\left(n^{\prime}-d\left(x_{i}\right)\right)\right\}$. The vote of $v_{i, j}^{A}$ is $\left(x_{i}^{\prime}, x_{i}^{2 j+1}, x_{i}^{2 j+2}\right)$.

- $N_{B}=\left\{v_{i, j}^{B}: 1 \leq i \leq n^{\prime}, 0 \leq j \leq\left(n^{\prime}-d\left(y_{i}\right)\right)\right\}$. The vote of $v_{i, j}^{B}$ is $\left(y_{i}^{\prime}, y_{i}^{2 j+1}, y_{i}^{2 j+2}\right)$.

- $N_{C}=\left\{v_{i, j}^{C}: 1 \leq i \leq n^{\prime}, 0 \leq j \leq\left(n^{\prime}-d\left(z_{i}\right)\right)\right\}$. The vote of $v_{i, j}^{C}$ is $\left(z_{i}^{\prime}, z_{i}^{2 j+1}, z_{i}^{2 j+2}\right)$.

- $N_{1}=\left\{v^{e}: e \in \mathcal{C}\right\}$. The vote of $v^{e}$ is $\left(x_{i}^{\prime}, y_{j}^{\prime}, z_{k}^{\prime}\right)$ where $e=\left(a_{i}, b_{j}, c_{k}\right) \in \mathcal{C}$.

- $N_{x^{*}}=\left\{v_{j}^{x^{*}}: 0 \leq j \leq n^{\prime}-1\right\}$. The vote of $v_{j}^{x^{*}}$ is $\left(x^{*}, x_{2 j+1}^{*}, x_{2 j+2}^{*}\right)$.

We claim that $I$ admits a perfect matching $M \subseteq \mathcal{C}$ if and only if $x^{*}$ becomes a possible cowinner by adding three new candidates.

Let $Y=\left\{y_{1}, y_{2}, y_{3}\right\}$ be the new candidates added. Since we cannot increase the score of $x^{*}$, we must decrease by one point the scores of candidates of $X^{\prime} \cup Y^{\prime} \cup Z^{\prime}$. Let us focus on candidates in $X^{\prime}$. In order to reduce the score of $x_{i}^{\prime}$, we must modify the votes of voters in $N_{1}$ or in $N_{A}$. By construction, each such voter must put $y_{1}, y_{2}, y_{3}$ in the first three positions (since in $N_{A}$ or from $(i i)$, candidates of $X^{\prime}$ are put in top position when they appear in the first three positions) and then, the score of each $y_{i}$ increases by 1 at each time. Since there are $n^{\prime}$ candidates in $X^{\prime}$, we deduce $S_{3}\left(y_{i}, P\right) \geq n^{\prime}$ for every $i=1,2,3$. On the other hand, if $x^{*}$ becomes a cowinner, $S_{3}\left(y_{i}, P\right) \leq S_{3}\left(x^{*}, P\right) \leq S_{3}\left(x^{*}, P_{X}\right)=n^{\prime}$ from $(i)$. Thus, $S_{3}\left(y_{i}, P\right)=n^{\prime}$ for every $i=1,2,3$ and there are exactly $n^{\prime}$ voters $N^{\prime}$ which put $y_{1}, y_{2}, y_{3}$ in the first three positions (for the remaining voters of $N \backslash N^{\prime}, y_{i}$ is ranked in position at least 4 for every $i=1,2,3$ ).

We claim that $N^{\prime} \subseteq N_{1}$. Otherwise, at least one voter of $N_{A}$ put $y_{1}, y_{2}, y_{3}$ in the first three positions. There remains at most $n^{\prime}-1$ voters of $N^{\prime}$ to decrease by 1 the score of candidates in $Y^{\prime}$. It is impossible because $\left|Y^{\prime}\right|=n^{\prime}$ and, from $(i i)$ and by construction of $N_{1}$, each candidate of $Y^{\prime}$ appears at most once in the first three positions for all voters. Finally, since the score of candidates in $Y^{\prime} \cup Z^{\prime}$ must also decrease by 1 , we deduce that $x^{*}$ is a possible cowinner iff $M=\left\{e \in \mathcal{C}: y_{1}, y_{2}, y_{3}\right.$ are in the first three positions for voter $\left.v_{e}\right\}$ is a perfect matching of $\mathcal{C}$.

\subsection{General case}

We finalize the study of the possible cowinner problem for $K$-approval with respect to candidate addition by showing that the problem is hard in any other case. For this we proceed in two steps: we first prove that for each $k \geq 3$, the problem $\operatorname{PCWNC}(k)$ for 3 -approval is NP-complete (Lemma 4). Next we prove that if the problem $\operatorname{PCWNC}(k)$ for $K$-approval is NP-complete then it is also the case for the problem $\operatorname{PCWNC}(k)$ for $(K+1)$-approval (Lemma 5).

Lemma 4 For all $k \geq 3$, the problem $\operatorname{PCWNC}(k)$ for 3-approval can be reduced in polynomial-time to the problem $\mathrm{PCWNC}(k+1)$ for 3-approval.

Proof: Let $\left\langle N, X, P_{X}, k, x^{*}\right\rangle$, where $P=P_{X}=\left\langle V_{1}, \ldots, V_{n}\right\rangle$, be an instance of PCWNC $(k)$ for 3-approval. Assume $S_{3}\left(x^{*}, P\right) \geq 1$ (otherwise, the problem is trivial). Consider the following instance $\left\langle N^{\prime}, X^{\prime}, Q_{X^{\prime}}, k+\right.$ $\left.1, x^{*}\right\rangle$ of the PCWNC $(k+1)$ for 3 -approval: 
- the set of candidates is $X^{\prime}=X \cup\{z\} \cup\left\{t_{i}^{1}, t_{i}^{2} \mid 1 \leq i \leq 2 S_{3}\left(x^{*}, P\right)\right\}$;

- there are $n+2 S_{3}\left(x^{*}, P\right)$ votes:

- for every vote $V_{j}$ in $P$ we have a vote $W_{j}$ in $Q$ whose first three candidates are the same as in $V_{j}$ and in the same order, and the other candidates are in an arbitrary order.

- for every $i=1, \ldots, 2 S_{3}\left(x^{*}, P\right)$, we have a vote $U_{i}$ in which the first 3 candidates are $t_{i}^{1}, t_{i}^{2}, z$, the remaining candidates being ranked arbitrarily.

Assume $x^{*}$ is a possible cowinner for $P=P_{X}$ (w.r.t. the addition of $k$ new candidates) and let $P^{\prime}$ be an extension of $P$ where $x^{*}$ is a cowinner. Let $Y=\left\{y_{1}, \ldots, y_{k}\right\}$ denote the new candidates for the instance $\left\langle N, X, P_{X}, k\right\rangle$, and $Y^{\prime}=\left\{y_{1}, \ldots, y_{k+1}\right\}$ the new candidates for the instance $\left\langle N^{\prime}, X^{\prime}, Q_{X^{\prime}}, k+1\right\rangle$. Consider the following extension $Q^{\prime}$ of $Q=Q_{X^{\prime}}$ : for every vote $V_{j}^{\prime}$ of $P^{\prime}$ we have a vote $W_{j}^{\prime}$ in $Q^{\prime}$ whose 3 first candidates are the same as in $V_{j}^{\prime}$ (and the remaining ones in an arbitrary order); and for every vote $U_{i}$ such that $1 \leq i \leq S_{3}\left(x^{*}, P\right)$ we have a vote $U_{i}^{\prime}$ whose first 3 candidates are $y_{k+1}, t_{i}^{1}, t_{i}^{2}$ and for every vote $U_{i}$ such that $S_{3}\left(x^{*}, P\right)+1 \leq i \leq 2 S_{3}\left(x^{*}, P\right)$, we have a vote $U_{i}^{\prime}$ whose first 3 candidates are $t_{i}^{1}, t_{i}^{2}, z$. It is easy to check that $Q^{\prime}$ is an extension of $Q$. The scores of all candidates in $X \cup Y$ are the same in $P^{\prime}$ and $Q^{\prime}$, while the score of each $t_{i}^{1}, t_{i}^{2}$ is 1 , the scores of $z$ and of $y_{k+1}$ are $S_{3}\left(x^{*}, P\right)$; therefore $x^{*}$ is a cowinner in $Q^{\prime}$ and a possible cowinner in $Q$.

Conversely, assume $x^{*}$ is a possible cowinner in $Q=Q_{X^{\prime}}$ and let $Q^{\prime}$ be an extension of $Q$ in which $x^{*}$ is a cowinner. We are now going to reason abut the number of occurrences of the new candidates $y_{1}, \ldots, y_{k+1}$ in the first three positions of the votes of $Q^{\prime}$. For the sake of notation, for any vote $V$ we denote $S_{3}\left(Y^{\prime}, V\right)=$ $\sum_{y \in Y^{\prime}} S_{3}\left(y^{\prime}, V\right)$ : in words, $S_{3}\left(Y^{\prime}, V\right)$ is the number of new candidates in the first three positions of $V$. Similarly, if $R$ is a profile, we denote $S_{3}\left(Y^{\prime}, R\right)=\sum_{y \in Y^{\prime}} S_{3}\left(y^{\prime}, R\right)$.

Without loss of generality, we assume that $S_{3}\left(x^{*}, Q^{\prime}\right)=S_{3}\left(x^{*}, Q\right)=S_{3}\left(x^{*}, P\right)$, since under 3-approval it is never beneficial to decrease the score of $x^{*}$ to make it a possible cowinner. We have $S_{3}\left(z, Q^{\prime}\right) \leq$ $S_{3}\left(x^{*}, Q^{\prime}\right)=S_{3}\left(x^{*}, P\right)$ and $S_{3}(z, Q)=2 S_{3}\left(x^{*}, P\right)$, therefore a new candidate must be put above $z$ in at least $S_{3}\left(x^{*}, P\right)$ votes $U_{i}^{\prime}$; therefore,

$$
\sum_{j=1}^{2 S_{3}\left(x^{*}, P\right)} S_{3}\left(Y^{\prime}, U_{i}\right) \geq S_{3}\left(x^{*}, P\right)
$$

Now, $S_{3}\left(Y^{\prime}, Q^{\prime}\right)=\sum_{i=1}^{n} S_{3}\left(Y^{\prime}, W_{i}\right)+\sum_{j=1}^{2 S_{3}\left(x^{*}, P\right)} S_{3}\left(Y^{\prime}, U_{i}\right)$, which together with (1) entails

$$
\sum_{i=1}^{n} S_{3}\left(Y^{\prime}, W_{i}\right) \leq S_{3}\left(Y^{\prime}, Q^{\prime}\right)-S_{3}\left(x^{*}, P\right)
$$

Now, $x^{*}$ is a cowinner in $Q^{\prime}$, therefore, for all $y_{j} \in Y^{\prime}$ we have $S_{3}\left(y_{j}, Q^{\prime}\right) \leq S_{3}\left(x^{*}, Q^{\prime}\right)=S_{3}\left(x^{*}, P\right)$, from which we get

$$
S_{3}\left(Y^{\prime}, Q^{\prime}\right) \leq(k+1) S_{3}\left(x^{*}, P\right)
$$

From (2) and (3) we get

$$
\sum_{i=1}^{n} S_{3}\left(Y^{\prime}, W_{i}\right) \leq k S_{3}\left(x^{*}, P\right)
$$


Now, consider the extension $P^{\prime}$ of $P$ built from the restriction of $Q^{\prime}$ to $\left\{W_{1}^{\prime}, \ldots, W_{n}^{\prime}\right\}$ by changing the candidates in $Y$ placed in the first three positions in such a way that each candidate appears at most in $S_{3}\left(x^{*}, P\right)$ votes, which is made possible by (4). We have:

- $S_{3}\left(x^{*}, P^{\prime}\right)=S_{3}\left(x^{*}, P^{\prime}\right)$;

- for each $y \in Y, S_{3}\left(y, P^{\prime}\right) \leq S_{3}\left(x^{*}, P\right)=S_{3}\left(x^{*}, P^{\prime}\right)$;

- for each $x \in X \backslash\left\{x^{*}\right\}, S_{3}\left(x, P^{\prime}\right)=S_{3}\left(x, Q^{\prime}\right)$; because $x^{*}$ is a possible cowinner in $Q^{\prime}$, we have $S_{3}\left(x, Q^{\prime}\right) \leq S_{3}\left(x^{*}, Q^{\prime}\right)=S_{3}\left(x^{*}, P\right)$, therefore, $S_{3}\left(x, P^{\prime}\right) \leq S_{3}\left(x^{*}, P\right)=S_{3}\left(x^{*}, P^{\prime}\right)$.

From this we conclude that $x^{*}$ is a possible cowinner in $P^{\prime}$.

Lemma 5 The problem $\operatorname{PcWNC}(k+1)$ for $K$-approval can be reduced in polynomial-time to the problem $\operatorname{PCWNC}(k)$ for $(K+1)$-approval.

Proof: Let $\left\langle N, X, P_{X}, k, x^{*}\right\rangle$ where $P_{X}=\left\langle V_{1}, \ldots, V_{n}\right\rangle$ be an instance of $\operatorname{PcWNC}(k)$ for $K$-approval. Consider the following instance $\left\langle N^{\prime}, X^{\prime}, R_{X^{\prime}}, k, x^{*}\right\rangle$ of the $\operatorname{PcWNC}(k)$ for $(K+1)$-approval:

- the set of candidates is $X^{\prime}=X \cup\left\{t_{i} \mid 1 \leq i \leq n\right\}$;

- for every vote $V_{i}$ in $P$ we have a vote $W_{i}$ in $R$ whose top candidate is $t_{i}$ and the candidates ranked in position 2 to $K+1$ are the candidates ranked in positions 1 to $K$ in $V_{i}$, the remaining candidates being ranked arbitrarily.

Assume $x^{*}$ is a possible cowinner for $P=P_{X}$ and let $P^{\prime}=\left\langle V_{1}^{\prime}, \ldots, V_{n}^{\prime}\right\rangle$ be an extension of $P$ where $x^{*}$ is a cowinner. Denote by $y_{1}, \ldots, y_{k}$ the new candidates. Consider the extension $R^{\prime}=\left\langle W_{1}^{\prime}, \ldots, W_{n}^{\prime}\right\rangle$ of $R=R_{X^{\prime}}$ where $W_{i}^{\prime}$ ranks $t_{i}$ first and then the candidates ranked in the first $K$ positions in $V_{i}^{\prime}$. For every $x \in X$ we have $S_{K+1}\left(x, R^{\prime}\right)=S_{K}\left(x, P^{\prime}\right)$; for every $i=1, \ldots, k$ we have $S_{K+1}\left(y_{i}, R^{\prime}\right)=S_{K}\left(y_{i}, P^{\prime}\right)$; and for every $j=1, \ldots, n$, we have $S_{K+1}\left(t_{j}, R^{\prime}\right)=1$. Therefore $x^{*}$ is a possible cowinner in $R^{\prime}$ and a possible cowinner in $R$.

Conversely, assume $x^{*}$ is a possible cowinner in $R=R_{X^{\prime}}$ and let $R^{\prime}=\left\langle W_{1}^{\prime}, \ldots, W_{n}^{\prime}\right\rangle$ be a completion of $R$ in which it is a possible cowinner. Since none of the $t_{i}$ threatens $x^{*}$, without loss of generality we assume $t_{i}$ still appears in the first $K+1$ positions of $W_{i}^{\prime}$-otherwise, change $W_{i}^{\prime}$ by moving $t_{i}$ to the top of $W_{i}^{\prime}$. Consider now the extension $P^{\prime}=\left\langle V_{1}^{\prime}, \ldots, V_{n}^{\prime}\right\rangle$ of $P=P_{X}$ where $V_{i}^{\prime}$ is obtained from $W_{i}^{\prime}$ by removing all the $t^{\prime}$ 's. Since exactly one $t_{i}$ appears in the first $K+1$ positions of $W_{i}^{\prime}$, the $K$ candidates approved in $V_{i}^{\prime}$ are exactly the $K+1$ candidates approved in $W_{i}^{\prime}$ minus $t_{i}$. From this we conclude that for every $x \in X$ we have $S_{K+1}\left(x, P^{\prime}\right)=S_{K}\left(x, R^{\prime}\right)$ and for every $i=1, \ldots, k$ we have $S_{K+1}\left(y_{i}, P^{\prime}\right)=S_{K}\left(y_{i}, R^{\prime}\right)$. Therefore $x^{*}$ is a possible cowinner in $P^{\prime}$ and a possible cowinner in $P$.

Proposition 7 Deciding whether a candidate is a possible cowinner for $K$-approval with respect to the addition of $k$ new candidates is NP-complete for each $(K, k)$ such that $K \geq 3$ and $k \geq 3$. 
Proof: Since deciding whether $x^{*}$ is a possible cowinner for 3 -approval with respect to the addition of 3 new candidates is NP-hard, using inductively the reductions of Lemma 4 and Lemma 5 shows that NP-hardness propagates to every $(K, k) \geq(3,3)$. Hence, the problem $\operatorname{PcWNC}(k)$ for $K$-approval is NP-complete for any fixed pair of values $K \geq 3$ and $k \geq 3$.

We summarize the results obtained in this Section by the following table:

\begin{tabular}{c|ccc} 
& $k=1$ & $k=2$ & $k \geq 3$ \\
\hline plurality & $\mathrm{P}$ (Prop. 1) & $\mathrm{P}$ (Prop. 1) & $\mathrm{P}$ (Prop. 1) \\
2-approval & $\mathrm{P}$ (Prop. 2) & $\mathrm{P}$ (Coro. 1) & $\mathrm{P}$ (Coro. 1) \\
$K$-approval, $K \geq 3$ & $\mathrm{P}$ (Prop. 2) & $\mathrm{P}$ (Prop. 5) & NP-complete (Prop. 7)
\end{tabular}

Observe that it would also be possible to address the $\operatorname{PCWNC}(k)$ problem (for $K \geq 3$ and $k \geq 3$ ) by working out a direct polynomial reduction from 3-DM, as done in Proposition 6 This would however result in a much less readable proof. One further interest of the proposed reduction is to show how it is possible to "neutralize" the (extended) power induced by adding more candidates by also adding one more (dummy) candidate in the initial instance. Intuitively, by setting the score of dummy candidate $t$ to $2 S_{K}\left(x^{*}, P\right)$, a single new candidate $y_{i}$ will have to be "consumed" to ensure that $t$ does not win. More generally, the same proof holds even if $K$ and $k$ depend on the instance (i.e. are not constant). If we allow $f(n)$ new candidates (where $f$ is polynomially bounded function) instead of $k$ a constant, the hardness result also holds (in the proof of Lemma 4 , we duplicate each vote $V f(n)$ times by adding candidates $z_{i}$ for $i=1, \ldots, f(n)$ instead of $z$ and we add dummy voters and candidates). Formally, we replace the construction given in Lemma 4 by:

- the set of candidates is $X^{\prime}=X \cup\left\{z_{1}, \ldots, z_{f(n)}\right\} \cup\left\{t_{i, \ell}^{1}, t_{i, \ell}^{2} \mid 1 \leq i \leq 2 S_{3}\left(x^{*}, P\right), 1 \leq \ell \leq f(n)\right\}$;

- there are $n+2 f(n) S_{3}\left(x^{*}, P\right)$ votes:

- for every vote $V_{j}$ in $P$ we have a vote $W_{j}$ in $Q$ whose first three candidates are the same as in $V_{j}$ and in the same order, and the other candidates are in an arbitrary order.

- for every $i=1, \ldots, 2 S_{3}\left(x^{*}, P\right)$ and $\ell=1, \ldots, f(n)$, we have a vote $U_{i, \ell}$ in which the first 3 candidates are $t_{i, \ell}^{1}, t_{i, \ell}^{2}, z_{\ell}$, the remaining candidates being ranked arbitrarily.

Finally, $Y^{\prime}=\left\{y_{1}, \ldots, y_{k+f(n)}\right\}$ are the new candidates.

Thus, using above construction, Lemma 4 and Proposition 6, we obtain that for any $\varepsilon \in(0 ; 1), \operatorname{PCWNC}(f(n))$ for 3-approval is an NP-complete problem where $f(n)=\Theta\left(|N|^{1-\varepsilon}\right)=\Theta\left(|X|^{1-\varepsilon}\right)$ (by setting $f(n)=|N|^{r}$ in the above construction where $r$ is a constant arbitrarily large). On the other hand, $\operatorname{PCWNC}(f(n))$ for $K$ approval is a problem which can be solved in polynomial time when $f(n)=K \cdot|N|$, i.e., when the number of new candidates is $K$ times the number of voters.

Note that some candidates (other than the new candidates) can be necessary cowinners with $K$-approval. Specifically, each candidate $x_{i}$ such that $S_{K-k}\left(P_{X}, x_{i}\right)=n$ is a necessary cowinner, since she is approved by all voters and there are not enough new candidates to push her (in at least one vote) out of the set of approved candidates. 


\section{Borda}

Let us now consider the Borda rule $\left(r_{B}\right)$. Characterizing possible Borda cowinners when adding candidates is easy due to the following lemma:

Lemma 6 Let $P_{X}$ be an $X$-profile where $X=\left\{x^{*}\right\} \cup\left\{x_{1}, \ldots, x_{p}\right\}$ and let $Y=\left\{y_{1}, \ldots, y_{k}\right\}$ be a set of $k$ new candidates. Let $r_{\vec{s}}$ be a scoring rule for $p+k$ candidate 1 defined by the vector $\vec{s}=\left\langle s_{1}, \ldots, s_{p}, \ldots, s_{p+k}\right\rangle$ such that $\left(s_{i}-s_{i+1}\right) \leq\left(s_{i+1}-s_{i+2}\right)$ for all $i . x^{*} \in X$ is a possible cowinner for $P_{X}$ w.r.t. the addition of $k$ new candidates for the scoring rule $r_{\vec{s}}(P)$ iff $x^{*} \in r(P)$ where $P$ is the profile on $X \cup Y$ obtained from $P_{X}$ by putting $y_{1}, \ldots, y_{k}$ right below $x^{*}$ (in arbitrary order) in every vote of $P_{X}$.

Proof: We show that it is never strictly better to put the new candidates anywhere but right below $x$ in the new profile. Let $P$ be an extension of $P_{X}$ in which $x^{*}$ is a cowinner, and assume there is a vote $V \in P$ and a new candidate $y$ such that either (i) $y \succ_{v} x^{*}$ or (ii) there exists at least one candidate $x^{\prime}$ such that $x^{*} \succ_{v} x^{\prime} \succ_{v} y$.

If we are in case (i), let us move $y$ right below $x^{*}$; let $V^{\prime}$ be the resulting vote, and $P^{\prime}$ the resulting profile. Obviously, $S_{\vec{s}}\left(y, P^{\prime}\right) \leq S_{\vec{s}}(y, P)$ and $S_{\vec{s}}\left(x^{*}, P^{\prime}\right) \geq S_{\vec{s}}\left(x^{*}, P\right)$, therefore $S_{\vec{s}}\left(x^{*}, P^{\prime}\right) \geq S_{\vec{s}}\left(y, P^{\prime}\right)$. For each candidate $z$ such that $y \succ_{v} z \succ_{v} x^{*}$, let $i$ be the rank of $z$ in $v$ and $j>i$ be the rank of $x^{*}$ in $v$. Then $\left(S_{\vec{s}}\left(z, P^{\prime}\right)-S_{\vec{s}}\left(x^{*}, P^{\prime}\right)\right)-\left(S_{\vec{s}}(z, P)-S_{\vec{s}}\left(x^{*}, P\right)\right)=\left(s_{i-1}-s_{j-1}\right)-\left(s_{i}-s_{j}\right)=\left(s_{i-1}-s_{i}\right)-\left(s_{j-1}-s_{j}\right) \leq 0$, therefore $S_{\vec{s}}\left(x^{*}, P^{\prime}\right) \geq S_{\vec{s}}\left(z, P^{\prime}\right)$. The scores of all other candidates are left unchanged, therefore $x^{*}$ is still a cowinner in $P^{\prime}$. By applying this process iteratively for all new candidates and in all votes until (i) no longer holds, we obtain a profile $Q$ in which $x^{*}$ is a cowinner, and such that $x^{*}$ is ranked above all new candidates in every vote.

Now, if (ii) holds for some new candidate $y$ and some vote $V$ of $Q$, then we move $y$ upwards, right below $x^{*}$; let $V^{\prime}$ be the resulting vote and $Q^{\prime}$ the resulting profile. The score of $y$ improves, but since $y$ is still ranked above all new candidates in every vote of $Q^{\prime}$, we have $S_{\vec{s}}\left(x^{*}, Q^{\prime}\right) \geq S_{\vec{s}}\left(y, Q^{\prime}\right)$. For each candidate $z \in X \cup Y \backslash\left\{x^{*}, y\right\}$ such that $x^{*} \succ_{v} z \succ_{v} y$ in vote $V, z$ moves down one position in $Q^{\prime}$, therefore $S_{\vec{s}}\left(z, Q^{\prime}\right) \leq S_{\vec{s}}\left(x^{\prime}, Q\right) \leq S_{\vec{s}}\left(x^{*}, Q\right)=S_{\vec{s}}\left(x^{*}, Q^{\prime}\right)$. The scores of all other candidates do not change, therefore $x^{*}$ is still a cowinner in $Q^{\prime}$. By applying this process iteratively and in all votes, until (ii) no longer holds, and we obtain a profile in which $x^{*}$ is a cowinner and neither (i) nor (ii) holds.

We conclude that $x^{*}$ is a possible cowinner for a profile if and only if it is a cowinner in an extension of the profile where all new candidates have been placed right below $x^{*}$.

In words, Lemma 6 applies to the rules where the difference of scores between successive ranks can only become smaller or remain constant as we come closer to the highest ranks. This condition is satisfied by Borda (but not by plurality), by veto, and by rules such as "lexicographic veto", where the scoring vector is $\left\langle M^{p}, M^{p}-M, M^{p}-M^{2}, \ldots, M^{p}-M^{p-1}, 0\right\rangle$ where $M>n$.

The following result then easily follows:

Proposition 8 Let $P_{X}$ be an $X$-profile where $X=\left\{x^{*}\right\} \cup\left\{x_{1}, \ldots, x_{p}\right\}$ and let $Y=\left\{y_{1}, \ldots, y_{k}\right\}$ be a set of $k$ new candidates. A candidate $x^{*}$ is a possible cowinner for Borda with respect to the addition of $k$ new

\footnotetext{
${ }^{1}$ In this lemma we do not have to deal with profiles with less than $p+k$ candidates, therefore it is not necessary to mention how $r_{s}$ is derived for fewer candidates than $p+k$.
} 
candidates if and only if

$$
k \geq \max _{z \in X \backslash\left\{x^{*}\right\}} \frac{S_{B}\left(z, P_{X}\right)-S_{B}\left(x^{*}, P_{X}\right)}{N_{P_{X}}\left(x^{*}, z\right)}
$$

Proof: By Lemma 6, $x^{*}$ a possible cowinner if and only if it is a cowinner in the $X \cup\left\{y_{1}, \ldots, y_{k}\right\}$-completion $P$ of $P_{X}$ where $y_{1}, \ldots, y_{k}$ are placed right below $x^{*}$, that is, if and only if $S_{B}\left(x^{*}, P\right)=S_{B}\left(x^{*}, P_{X}\right)+k n$. Now, for each vote, all candidates in $X \backslash\left\{x^{*}\right\}$ ranked above $x^{*}$ get $k$ additional points in the extended vote, while those ranked below $x^{*}$ keep the same score. Hence, for every $z \in X \backslash\left\{x^{*}\right\}$ we have $S_{B}(z, P)=$ $S_{B}\left(z, P_{X}\right)+k\left(n-N_{P_{X}}\left(x^{*}, z\right)\right)$, therefore, $x^{*}$ is a cowinner in $P$ if and only if $S_{B}\left(x^{*}, P_{X}\right)+k n \geq$ $S_{B}\left(z, P_{X}\right)+k\left(n-N_{P_{X}}\left(x^{*}, z\right)\right)$, which is equivalent to $k \geq\left[S_{B}\left(z, P_{X}\right)-S_{B}\left(x^{*}, P_{X}\right)\right] / N_{P_{X}}\left(x^{*}, z\right)$. (We recall that $N_{P_{X}}\left(x^{*}, z\right)$ stands for the number of votes in $P_{X}$ ranking $x^{*}$ above $z$ ).

In words, checking whether $x^{*}$ is a possible cowinner boils down to checking, for each other candidate $z$, whether there are enough votes where $x^{*}$ is preferred to $z$ to compensate for the score difference with this candidate. This means that possible cowinners with respect to adding any number of new candidates can be computed in polynomial time for Borda, and more generally for any rule satisfying the conditions of Lemma 6 Note that computing possible winners for Borda is NP-hard [30], therefore, the restriction of the problem to candidate addition induces a complexity reduction.

Example 3 Take $X=\{a, b, c, d\}, n=4$, and $P_{X}=\langle$ bacd, bacd,bacd,dacb $\rangle$. The Borda scores in $P_{X}$ are $S_{B}\left(a, P_{X}\right)=8, S_{B}\left(b, P_{X}\right)=9, S_{B}\left(c, P_{X}\right)=3$, and $S_{B}\left(d, P_{X}\right)=4$, while $N(a, b)=1, N(a, c)=4$, $N(a, d)=3, N(b, c)=3, N(b, d)=3, N(c, d)=3$, and for all $x, y, N(x, y)=4-N(y, x)$. Let $\delta(x, z)=S_{B}\left(z, P_{X}\right)-S_{B}\left(x, P_{X}\right) / N_{P_{X}}(x, z)$. The following matrix gives the values of $\delta(x, z)$ for the possible pairs of distinct candidates (for the sake of readability, non-positive values are denoted by $\leq 0$ ).

\begin{tabular}{|c|cccc|c|}
\hline$\delta(x, z)$ & $a$ & $b$ & $c$ & $d$ & $\max$ \\
\hline$a$ & - & 1 & $\leq 0$ & $\leq 0$ & 1 \\
$b$ & $\leq 0$ & - & $\leq 0$ & $\leq 0$ & $\leq 0$ \\
$c$ & $+\infty$ & 5 & - & $\leq 0$ & $+\infty$ \\
$d$ & 5 & 6 & 1 & - & 6 \\
\hline
\end{tabular}

Applying Proposition 8 $b$ is a possible cowinner whatever the value of $k$, a is a possible cowinner if and only if $k \geq 1, d$ is a possible cowinner if and only if $k \geq 6$, c is not a possible cowinner whatever the value of $k^{2}$. Note that for $k \geq 6$, d is a possible cowinner whereas $c$ is not, although c has a higher Borda score than $d$ in $P_{X}$.

\section{Hardness with a single new candidate}

Even though we have seen that the possible cowinner problem can be NP-hard for some scoring rules, NPhardness required the addition of several new candidates. We now show that there exists a scoring rule for which the possible cowinner problem is NP-hard with respect to the addition of one new candidate.

\footnotetext{
${ }^{2}$ This is so because $c$ is always ranked below $a$. We make this intuition clear in Section 7
} 
The scoring rule we use is very simple: it allows each voter to approve exactly 3 candidates, and offers 3 different levels of approval (assigning respectively 3,2,1 points to the three preferred candidates). Let $r_{\Delta}$ be the scoring rule defined by the vector $\vec{s}=\langle 3,2,1,0, \ldots, 0\rangle$ with $m-30$ 's completing the vector.

Proposition 9 Deciding if $x^{*}$ is a possible cowinner for $r_{\Delta}$ with respect to the addition of one candidate is NP-complete.

Proof: This problem is clearly in NP. The hardness proof is quite similar to that of Proposition 6 Let $I=$ $(\mathcal{C}, A \times B \times C)$ be an instance of 3 -DM with $n^{\prime} \geq 5$ and $\forall z \in A \cup B \cup C, d(z) \in\{2,3\}$. From $I$, we build an instance $\left\langle N, X, P_{X}, 1, x^{*}\right\rangle$ of the PcWNC problem as follows. The set $X$ of candidates contains $x^{*}$, $X_{1}=\left\{x_{i}^{\prime}, y_{i}^{\prime}, z_{i}^{\prime}: 1 \leq i \leq n^{\prime}\right\}$ where $x_{i}^{\prime}, y_{i}^{\prime}, z_{i}^{\prime}$ correspond to elements of $A, B$ and $C$ respectively and a set $X_{2}$ of dummy candidates. The set $N$ of voters contains $N_{1}=\left\{v^{e}: e \in \mathcal{C}\right\}$ and a set $N_{2}$ of dummy voters. For each voter $v_{i} \in N$, we only indicate the vote for the first three candidates. So, the vote $V_{i}=\left(t_{1}, t_{2}, t_{3}\right)$ means that candidate $t_{i}$ receives $4-i$ points. The vote $V_{e}$ of voter $v^{e}$ is $\left(x_{i}^{\prime}, y_{j}^{\prime}, z_{k}^{\prime}\right)$ where $e=\left(a_{i}, b_{j}, c_{k}\right) \in \mathcal{C}$. The preferences of dummy voters are such that $(a)$ the score of the candidates in $X$ satisfies $\forall x \in X_{1}$, $S_{\vec{s}}\left(x, P_{X}\right)=3 n^{\prime}+1, S_{\vec{s}}\left(x^{*}, P_{X}\right)=3 n^{\prime}$ and $\forall x \in X_{2}, S_{\vec{s}}\left(x, P_{X}\right) \leq 3$ and $(b)$ each voter in $N_{2}$ ranks at most one candidate of $\left\{x_{i}^{\prime}, y_{i}^{\prime}, z_{i}^{\prime}: 1 \leq i \leq n^{\prime}\right\}$ in the first three positions, and if he ranks one in second position, then $x^{*}$ occurs in third position.

Formally, the instance of the PcWNC problem is built as follows. The set of voters is $N=N_{1} \cup N_{2}$ where $N_{1}=\left\{v^{e}: e \in \mathcal{C}\right\}$ and $N_{2}=N_{A} \cup N_{B} \cup N_{C} \cup N_{x^{*}}$, the set of candidates is $X=X_{1} \cup X_{2} \cup\left\{x^{*}\right\}$ where $X_{1}=X^{\prime} \cup Y^{\prime} \cup Z^{\prime}$ with $X^{\prime}=\left\{x_{1}^{\prime}, \ldots, x_{n^{\prime}}^{\prime}\right\}, Y^{\prime}=\left\{y_{1}^{\prime}, \ldots, y_{n^{\prime}}^{\prime}\right\}, Z^{\prime}=\left\{z_{1}^{\prime}, \ldots, z_{n^{\prime}}^{\prime}\right\}$ and $X_{2}=X_{A} \cup X_{B} \cup X_{C} \cup X_{x^{*}}$. These sets are defined as follows:

- $X_{A}=\left\{x_{i}^{j}: 1 \leq i \leq n^{\prime}, 1 \leq j \leq 2\left(n^{\prime}-d\left(a_{i}\right)\right)\right\}$.

- $X_{B}=\left\{y_{i}^{j}: 1 \leq i \leq n^{\prime}, 1 \leq j \leq 2\left(3 n^{\prime}-2 d\left(b_{i}\right)+1\right)\right\}$.

- $X_{C}=\left\{z_{i}^{j}: 1 \leq i \leq n^{\prime}, 1 \leq j \leq 2\left(3 n^{\prime}-d\left(c_{i}\right)+1\right)\right\}$.

- $X_{x^{*}}=\left\{x_{i}^{*}: i=1 \leq i \leq 2 n^{\prime}\right\}$.

The set of all $X$-votes $\mathcal{P}_{X}$ is given by:

- $N_{A}=\left\{v_{i, j}^{A}: 1 \leq i \leq n^{\prime}, 0 \leq j \leq\left(n^{\prime}-d\left(a_{i}\right)-2\right)\right\} \cup\left\{v_{i}^{A, j}: 1 \leq i \leq n^{\prime}, j=1,2\right\}$. The vote $V_{i, j}^{A}$ of $v_{i, j}^{A}$ is $V_{i, j}^{A}=\left(x_{i}^{\prime}, x_{i}^{2 j+1}, x_{i}^{2 j+2}\right)$. Note that $n^{\prime}-d\left(a_{i}\right)-2 \geq 0$. The vote of $v_{i}^{A, j}$ is $V_{i}^{A, j}=\left(x_{i}^{2\left(n^{\prime}-d\left(a_{i}\right)-1\right)+j}, x_{i}^{\prime}, x^{*}\right)$.

- $N_{B}=\left\{v_{i, j}^{B}: 1 \leq i \leq n^{\prime}, 0 \leq j \leq 3 n^{\prime}-2 d\left(b_{i}\right)\right\}$. The vote of $v_{i, j}^{B}$ is $V_{i, j}^{B}=\left(y_{i}^{2 j+1}, y_{i}^{2 j+2}, y_{i}^{\prime}\right)$.

- $N_{C}=\left\{v_{i, j}^{C}: 1 \leq i \leq n^{\prime}, 0 \leq j \leq 3 n^{\prime}-d\left(c_{i}\right)\right\}$. The vote of $v_{i, j}^{C}$ is $V_{i, j}^{C}=\left(z_{i}^{2 j+1}, z_{i}^{2 j+2}, z_{i}^{\prime}\right)$.

- $N_{x^{*}}=\left\{v_{j}^{x^{*}}: 0 \leq j \leq n^{\prime}-1\right\}$. The vote of $v_{j}^{x^{*}}$ is $V_{j}^{x^{*}}=\left(v_{2 j+1}^{x^{*}}, v_{2 j+2}^{x^{*}}, x^{*}\right)$. Note that $n^{\prime}-1 \geq 0$.

- $N_{1}=\left\{v^{e}: e \in \mathcal{C}\right\}$. The vote of $v^{e}$ is $V_{e}=\left(x_{i}^{\prime}, y_{j}^{\prime}, z_{k}^{\prime}\right)$ where $e=\left(a_{i}, b_{j}, c_{k}\right) \in \mathcal{C}$.

We claim that $I$ admits a perfect matching $M \subseteq \mathcal{C}$ if and only if $x^{*}$ becomes a possible cowinner by adding a new candidate $y_{1}$. Observe that the scores of the candidates in $X$ satisfy: 
(i) $\forall x \in X_{1}, S_{\vec{s}}\left(x, P_{X}\right)=3 n^{\prime}+1$.

(ii) $S_{\vec{s}}\left(x^{*}, P_{X}\right)=3 n^{\prime}$.

(iii) $\forall x \in X_{2}, S_{\vec{s}}\left(x, P_{X}\right) \leq 3$.

Items $(i),(i i)$ and $(i i i)$ correspond to the conditions $(a)$ and $(b)$ described previously. For instance, each candidate $x_{i}^{\prime}$ from $X_{1}$ gets respectively 3,3 , and 2 points from the votes $V_{e}, V_{i, j}^{A}$, and $V_{i}^{A, j}$, summing up to $3 d\left(a_{i}\right)+3\left(n^{\prime}-d\left(a_{i}\right)-1\right)+2=3 n^{\prime}+1$. The reader can easily check that the conditions also hold for all other candidates.

Let $y_{1}$ be the new candidate. By construction of this scoring rule, we must decrease the score of candidates in $X$ which dominate the score of $x^{*}$, that is the candidates of $X_{1}$ using $(i)$ and (iii).

Let $P^{\prime}$ be a $X \cup\left\{y_{1}\right\}$-profile such that $x^{*}$ is a cowinner. Let us focus on candidates in $X^{\prime}$. In order to reduce the score of $x_{i}^{\prime}$ by 1 , we must modify the preference for at least one voter $v^{e}$ or $v_{i, j}^{A}$ or $v_{i}^{A, j}$. If we modify it for some voter in $v_{i}^{A, j}$, then the score of $x_{i}^{\prime}$ (with respect to $v_{i}^{A, j}$ ) decreases by one if and only if the score of $x^{*}$ (with respect to $v_{i}^{A, j}$ ) also decreases by one. In conclusion, we must modify the preference of $x_{i}^{\prime}$ for at least one voter $v^{e}$ or $v_{i, j}^{A}$. By construction, each such voter must put $y_{1}$ in top position and then, the score of $y_{1}$ increases by 3 at each time. Since there are $n^{\prime}$ candidates in $X^{\prime}$, we deduce $S_{\vec{s}^{\prime}}\left(y_{1}, P^{\prime}\right) \geq 3 n^{\prime}$; From above remark, we also get $S_{\vec{s}^{\prime}}\left(x^{*}, P^{\prime}\right) \leq S_{\vec{s}^{\prime}}\left(x^{*}, P_{X}\right)=3 n^{\prime}$. Thus for each $i \in\left\{1, \ldots, n^{\prime}\right\}$, exactly one voter among those of $v^{e}$ or $v_{i, j}^{A}$ must put candidate $y_{1}$ in top position. Finally, if it is one voter $v_{i, j}^{A}$, then we deduce $S_{\overrightarrow{s^{\prime}}}\left(y_{1}, P^{\prime}\right)>3 n$ because the score of $Y^{\prime} \cup Z^{\prime}$ must also decrease, which is not possible since $y_{1}$ will then win.

Following a line of reasoning similar to the one developed in the proof of Proposition 6 we conclude that for each $i \in\left\{1, \ldots, n^{\prime}\right\}$, exactly one voter among those of $v^{e}$ must put candidate $y_{1}$ in top position. Since the score of $Y^{\prime} \cup Z^{\prime}$ must also decrease by 1 , we deduce that $x^{*}$ is a possible cowinner if and only if $M=\{e \in \mathcal{C}$ : $y_{1}$ is in top position in vote $\left.V_{e}\right\}$ is a perfect matching of $\mathcal{C}$ (for the remaining voters, $y_{1}$ is put in last position).

This rule shows that it may be difficult to identify possible cowinners with a single additional candidate. Giving a characterization of all rules possessing this property is an open problem.

\section{Related work}

\subsection{The possible winner problem}

The possible winner problem was introduced in [22]: given an incomplete profile $P=\left\langle V_{1}, \ldots, V_{n}\right\rangle$ where each $V_{i}$ is a partial order over the set of candidates $X, x$ is a possible winner for $P$ given a voting rule $r$ if there exists a complete extension $P^{\prime}=\left\langle V_{1}^{\prime}, \ldots, V_{n}^{\prime}\right\rangle$ of $P$, where each $V_{i}^{\prime}$ is a linear order on $X$ extending $V_{i}$, such that $r\left(P^{\prime}\right)=x$. Possible winners are defined in a similar way for a voting correspondence $C$, in which case we say that $x$ is a possible cowinner if there exists an extension $P^{\prime}$ of $P$ such that $x \in C\left(P^{\prime}\right)$. Clearly, the possible winner problem defined in this paper is a restriction of the general possible winner problem to the following set of incomplete profiles:

(Restr) there exists $X^{\prime} \subseteq X$ such that for every $i, V_{i}$ is a linear order on $X^{\prime}$ 
As an immediate corollary, the complexity of the possible (co)winner problem with respect to candidate addition is at most as difficult as that of the general problem. This raises the question whether (Restr) leads to a complexity reduction for the scoring rules we have considered here.

The possible (co)winner problem for scoring rules has received a significant amount of attention in the last years. Xia and Conitzer [30] proved that the problem was NP-complete for the Borda rule, and more generally for scoring rules whose scoring vector contains four consecutive, equally decreasing values, followed by another strictly decreasing value. Betzler and Dorn [5] went further by showing that NP-completeness holds more generally for all pure 3 scoring rules, except plurality, veto, and scoring rules whose vector $s^{m}$ is of the form $s^{m}=\langle 2,1, \ldots, 1,0\rangle$ for large enough values of $m$. The issue was finally closed by Baumeister and Rothe [3], who showed that the problem for $s^{m}=\langle 2,1, \ldots, 1,0\rangle$ is NP-complete as well. These results compare to ours in the following way: all our NP-hardness results strengthen the known NP-hardness results for the general possible winner problem, while our polynomiality results show a complexity reduction induced by (Restr).

Two recent papers give results about the PCWNC problem for other voting rules. Xia et al. [33] give results about the possible (co)winner with new candidates for other voting rules: they showed that PWNC and

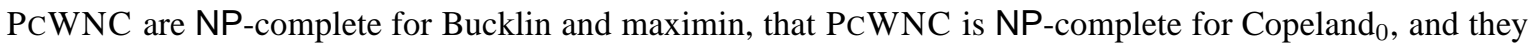
give several results for approval voting, depending on how the extension a vote is defined. Baumeister et al. [4] generalize our Proposition 9 by showing that the PCWNC problem is NP-complete for any pure scoring rule of the form $\left\langle\alpha_{1}, \alpha_{2}, 1 ; 0, \ldots, 0\right\rangle$; they also give NP-completeness results for plurality and 2-approval when voters are weighted.

Results about the PCWNC known so far (except our Proposition 9 and its generalization by [4]) are summarized in the following table. For the sake of completeness, we also mention the complexity of the other prominent subproblem of the possible cowinner problem, namely unweighted coalitional manipulation.

\begin{tabular}{l|l|l|l|} 
& general problem & candidate addition & manipulation \\
\hline plurality and veto & $\mathrm{P}$ & $\mathrm{P}$ (Prop. 1] & $\mathrm{P}$ \\
Borda & NP-complete [30] & $\mathrm{P}$ (Prop. 8] & NP-complete [7, 12] \\
2-approval & NP-complete [5] & $\mathrm{P}$ (Coro. 1] & $\mathrm{P}$ \\
$K$-approval, $K \geq 3$ & NP-complete [5] & NP-complete (Prop. 6] & $\mathrm{P}$ \\
Bucklin & NP-complete [30] & NP-complete [33] & $\mathrm{P}$ [32] \\
maximin $_{\text {Copeland }}$ & NP-complete [30] & NP-complete [33] & NP-complete [32] \\
& NP-complete [30] & NP-complete [33] & NP-complete [16]
\end{tabular}

Another interesting line of work is the parameterized complexity of the possible winner problem for scoring rules, which has been investigated in [6]. Among other results, they show that for all scoring rules, the problem is fixed-parameter tractable with respect to the number of candidates (in particular, when the number of candidates is bounded by a constant, the problem becomes polynomial-time solvable). This polynomiality result clearly holds in the possible winner problem with respect to candidate addition, with some caution: the number of candidates here is the total number of candidates (the initial ones plus the new ones); this result has practical impact in some situations mentioned in the introduction, such as finding a date for a meeting, where the number of candidates is typically low.

\footnotetext{
${ }^{3} \mathrm{~A}$ (family of) scoring rules $\left(r_{m}\right)_{m \geq 1}$ is pure if for each $m$, the scoring vector for $m+1$ candidates is obtained from the scoring vector for $m$ candidates by inserting an additional score at an arbitrary position. All interesting families of scoring rules are pure; this is in particular the case for $K$-approval and Borda.
} 
We end this subsection by mentioning other works on the possible winner problem and its variants and subproblems, that are less directly connected to our results. The possible winner problem has also been studied from the probabilistic point of view by Bachrach et al. [1], where the aim is to count the number of extensions in which a given candidate is the winner. Such a probabilistic analysis is highly relevant in candidate-adding situations: given $P_{X}$, a number $k$ of new candidates, and a prior probability distribution on votes, computing the probability that a given candidate $x \in X$ will be the winner, or that one of the initial (resp. new) candidates will be the winner, is extremely interesting 4

\subsection{Control via adding candidates}

The possible winners with respect to the addition of candidates is highly reminiscent of constructive control by the chair via adding candidates - this problem first appeared in [2] and was later studied in more depth for many voting rules, see e.g., [21, 17]. However, even if a voting situation where new candidates are added looks similar to an instance of constructive control by adding candidates, these problems differ significantly. In control via adding candidates, the input consists of a set of candidates $X$, a set of "spoiler" candidates $Y$, and a full profile $P_{X \cup Y}$ : the chair knows how the voters would vote on the new candidates; the problem is to determine whether a given candidate $x^{*}$ can be made a winner by adding at most $k \leq|Y|$ candidates from $Y$. In the possible winner problem with respect to candidate addition, we have to take into account all possible ways for voters to rank the new candidates. In spite of their significant differences, there is a straightforward connection between these problems: if an instance $\left\langle N, X, P_{X \cup Y}, x^{*}, k\right\rangle$ of control via adding candidates is positive, then $x^{*}$ is a possible winner in $P_{X}$ with respect to the addition of $k$ new candidates (the voting rule being the same in both problems).

Bartholdi et al. [2] noted that a voting rule is immune to control by adding candidates as soon as it satisfies the Weak Axiom of Revealed Preference (WARP), which requires that the winner among a set of candidates $W$ to be the winner among every subset of candidates to which he belongs [27]; formally: for any $Z \subseteq W$, if $r\left(P_{W}\right) \in Z$ then $r\left(P_{Z}\right)=r\left(P_{W}\right)$. This property can be used in a similar way for the possible winner problem with respect to candidate addition. Obviously, if the voting rule $r$ satisfies WARP, then any possible winner from $X$ is a winner for the current profile $P_{X} 5$. Unfortunately, this social-choice theoretic property is very strong: [13] show that a voting rule satisfies this property (there, it is called candidate stability) and unanimity if and only if it is dictatorial.

\subsection{Cloning}

Finally, the possible winner problem via candidate addition is closely related to manipulation by candidate cloning. Independence of clones was first studied in [28], further studied in [24, 23], and a variant of this

\footnotetext{
${ }^{4}$ Note that if the voting rule is insensitive to the identity of candidates (i.e. neutral), then although the prior probability that one of the $k$ new candidates will be a cowinner under the impartial culture assumption is at least $\frac{k}{|X|+k}$, this is no longer the case when $P_{X}$ is known: for instance, let us use plurality and consider the profile $P_{X}=\langle a b, a b, a b\rangle$, and let the number of new candidates be one. For a third candidate to be a cowinner, he either needs to be placed first in all three votes (which occurs with probability $\frac{1}{27}$ ), or to be placed first in two votes, but not in the third vote (which occurs with probability $\frac{6}{27}$ ); therefore the probability that the new candidate is a cowinner in the completed profile is only $\frac{7}{27}$.

${ }^{5}$ In order for the converse to hold, we must add one more condition, such as consensus (a Pareto-dominated candidate cannot be elected). Then, if the winner for the current profile $P_{X}$ is $x$, by ranking all new candidates at the bottom of all votes, none of them can be the winner in $P_{X \cup Y}$, and by WARP, no candidate $x^{\prime} \in X \backslash\{x\}$ can either, therefore $x$ is a possible winner for $P_{X}$ with respect to candidate addition.
} 
property was recently considered from the computational point of view in [14]. The main difference between $x$ being a possible winner with respect to candidate addition and the existence of a candidate cloning strategy so that $x$ or one of its clones becomes the winner, as in [14], is that candidate cloning requires a candidate and its clones to be contiguous in all votes. In other terms, whereas our problem considers the introduction of genuinely new candidates, cloning merely introduces copies of existing ones.

The complexity of this problem is considered by Elkind et al. [14] for several voting rules. Although the proposed model allows for the possibility of having a bounded number of new clones (via a notion of cost), most of their results focus on the case of unboundedly many clones. Therefore, to be able to compare their results with ours, we should first say something about the variant of the possible winner problem with respect to candidate addition, when the number of new candidates is not known beforehand and can be arbitrarily large. The definitions of voting situations and possible winners are straightforward adaptations of Definitions 1 and 2, a voting situation is now a triple $\Sigma=\left\langle N, X, P_{X}\right\rangle$ and $x^{*}$ is a possible cowinner with respect to $\Sigma$ and $r$ if there exists an integer $k$ and a set $Y$ of cardinality $k$ such that there is a $(X \cup Y)$-profile $P$ extending $P_{X}$ such that $x^{*} \in r(P)$. We now give a necessary and sufficient condition for a candidate to be a possible winner, for a class of scoring rules including the Borda rule.

Proposition 10 Let $\mathcal{S}$ be a collection of scoring vectors $\left(s^{m}\right), m \geq 1$, such that

- for every $p,\left(s_{j}^{m}\right), 1 \leq j \leq m$ is strictly decreasing;

- for all $j, j^{\prime} \in \mathbb{N},(1) \lim _{m \rightarrow \infty} \frac{s_{j}^{m}-s_{j^{\prime}}^{m}}{s_{1}}=0$ and (2) $\lim _{m \rightarrow \infty} \frac{s_{j}^{m}-s_{m-j^{\prime}}^{m}}{s_{1}}=1$.

Then, $x^{*}$ is a possible winner w.r.t. $\left\langle N, X, P_{X},+\infty\right\rangle$ if and only if it is undominated in $P_{X}$.

Proof: First, suppose $x^{*}$ is undominated in $P_{X}$. For any candidate $x_{i} \neq x^{*}$, define $\Delta_{i}^{v}$ as the difference between the score of $x^{*}$ and the score of $x_{i}$, divided by $s_{1}$, in the vote $v$. As in the construction of Lemma 1, put $k$ new candidates right below $x^{*}$ in every vote, and let $P^{\prime}$ be the resulting profile. As the value of $k$ grows, for any vote $v$ ranking candidate $x^{*}$ below $x_{i}$, the value of $\Delta_{i}^{v}$ will tend towards 0 (by condition 1). Also, condition 2 ensures that for each vote $v$ ranking $x^{*}$ above $x_{i}$, the value of $\Delta_{i}^{v}$ tends towards 1 . Because $x^{*}$ is undominated, such votes always exist for every candidate $x_{i} \neq x^{*}$. Therefore, when $k$ grows, $\sum_{v \in P^{\prime}} \Delta_{i}^{v}$ tends towards the number of votes ranking $x^{*}$ above $x_{i}$, which is at least 1 . This implies that the score of $x^{*}$ will be eventually larger than the score of $x_{i}$, and this is true for every $x_{i} \neq x^{*}$, therefore $x^{*}$ will eventually become the winner as $k$ grows. Conversely, suppose $x^{*}$ is dominated by some candidate $x_{i}$. Because the scores $\left(s_{j}^{m}\right), 1 \leq j \leq m$ are strictly decreasing, the score of $x$ will always remain strictly below the score of $x_{i}$ in the completion of the profile, hence $x^{*}$ is not a possible cowinner.

Clearly, this large class of voting rules includes Borda, since it satisfies the conditions of Proposition 10 However, it does not include plurality, and more generally $K$-approval, which violate condition (1). Still, a very simple condition can be stated for $K$-approval: a candidate is a possible winner as soon as it is approved at least once.

Proposition 11 When $r$ is $K$-approval, $x^{*}$ is a possible winner w.r.t. the addition of an unbounded number of new candidates if and only if $S_{K}\left(x^{*}, P_{X}\right) \geq 1$.

\footnotetext{
${ }^{6}$ We recall that candidate $x$ dominates candidate $x^{\prime}$ if every voter ranks $x$ above $x^{\prime}$, and that a candidate is undominated if no other candidate dominates it.
} 
Proof: The condition is obviously necessary. Suppose the condition holds on a given profile. We extend this profile by taking a set of new candidates $y_{i j}$ where $1 \leq i \leq n$ and $1 \leq j \leq K$. Consider the $i$-th vote: if $x^{*}$ is ranked in one the top $k$ positions, put all new candidates at the bottom of the vote. Otherwise, introduce the new candidates $\left\{y_{i 1}, \ldots, y_{i K}\right\}$ at the top of the vote, and all other new candidates at the bottom. The score of the new candidates is at most 1 , while that of $x_{i} \neq x^{*}$ is at most that of $x^{*}$ (which is unchanged).

Note that for $K \geq 2$ this condition does not imply that the candidate is undominated (nor vice-versa). It does obviously when $K=1$, i.e., for plurality.

Let us see now how the above results relate to those in [14]. We first note that in the case of the Borda rule we have the same condition. Indeed one sees intuitively that Lemma 6 tells us that for some voting rules (including Borda), introducing new candidates in a contiguous manner, as with cloning, is the best thing to do. For plurality, again the condition is similar in both cases. However, for $K$-approval as soon as $K>1$, the problem becomes hard in the cloning setting whereas it is easy in our setting with an unbounded number of new candidates.

\section{Conclusion}

In this paper we have considered voting situations where new candidates may show up during the process. This problem increasingly occurs in our societies, as many votes now take place online (through dedicated platforms, or simply by email exchange) during an extended period of time.

We have identified the computational complexity of computing possible winners for some scoring rules. Some of them allow polynomial algorithms for the problem (e.g. plurality, 2-approval, Borda, veto) regardless of the (fixed) number of new candidates showing up. For the rules of the $K$-approval family, when $K \geq 3$, the problem remains polynomial only if the number of new candidates is at most 2 . Finally, we have exhibited a simple rule where the problem is hard for a single new candidate.

The results address the problem of making some designated candidate a cowinner, which is similar to $x$ being unique winner under the assumption of the most favourable tie-breaking. In the other extreme case (if we want $x$ to be a strict winner, i.e., to win regardless of the tie-breaking rule), the results are easily adapted: for instance, the inequalities in Proposition 11 and 8 become strict. For $K$-approval, the first condition of Proposition 2 becomes strict but the second one should now read $S_{K}\left(P_{X}, x\right) \geq \sum_{x_{i} \in X} \max \left(0, S_{K}\left(P_{X}, x_{i}\right)-\right.$ $\left.S_{K}\left(P_{X}, x\right)+1\right)$. As for veto, all other initial candidates need to be vetoed at least once. The hardness proofs can also be readily adapted to the unique winner setting. A more general treatment would require cumbersome expressions, and is also somewhat problematic since the identities of the new candidates are not known anyway (making it difficult to specify easily a tie-breaking rule on these candidates).

As for future work, a first direction to follow would be to try to obtain more general results for scoring rules, as those obtained by Betzler and Dorn [5] for the general version of the possible winner problem. Extending the study to other families of voting rules, such as rules based on the majority graph, is also worth investigating.

Of course, identifying possible winners is not the end of the story. In practice, as mentioned earlier, one may for instance also be interested in a refinement of this notion: knowing how likely it is that a given candidate will win. Another interesting issue consists in designing elicitation protocols when the preferences about the 'old' 
candidates are already known. In this case, a trade-off occurs between the storage cost and communication cost, since keeping track of more information is likely to help reduce the burden of elicitation.

Acknowledgements. We are very much indebted to the reviewers of previous versions of this paper for their extremely detailed and relevant comments.

\section{References}

[1] Y. Bachrach, N. Betzler, and P. Faliszewski. Probabilistic possible-winner determination. In Proceedings of AAAI-10, pages 697-702, 2010.

[2] J. Bartholdi, C. Tovey, and M. Trick. How hard is it to control an election? Social Choice and Welfare, 16(8-9):27-40, 1992.

[3] D. Baumeister and J. Rothe. Taking the final step to a full dichotomy of the possible winner problem in pure scoring rules. In Proceedings of ECAI 2010, pages 1019-1020, 2010.

[4] D. Baumeister, M. Roos and J. Rothe. Computational complexity of two variants of the possible winner problem. Proceedings of AAMAS-11, pages 853-860, 2011.

[5] N. Betzler and B. Dorn. Towards a dichotomy of finding possible winners in elections based on scoring rules. In Proceedings of MFCS 2009, volume 5734 of Lecture Notes in Computer Science, pages 124-136. Springer, 2009.

[6] N. Betzler, S. Hemmann, and R. Niedermeier. A multivariate complexity analysis of determining possible winners given incomplete votes. In Proceedings of IJCAI-09, pages 53-58, 2009.

[7] N. Betzler, R. Niedermeier and G. Woeginger. Unweighted Coalitional Manipulation Under the Borda Rule is NP-Hard In Proceedings of IJCAI-11, 55-60, 2011.

[8] Y. Chevaleyre, J. Lang, N. Maudet, and G. Ravilly-Abadie. Compiling the votes of a subelectorate. In Proceedings of IJCAI-09, pages 97-102, 2009.

[9] V. Conitzer and T. Sandholm. Complexity of manipulating elections with few candidates. In Proceedings of AAAI-02, pages 314-319, 2002.

[10] V. Conitzer and T. Sandholm. Vote elicitation: complexity and strategy-proofness. In Proceedings of AAAI-02, pages 392-397, 2002.

[11] V. Conitzer, T. Sandholm and J. Lang. When are elections with few candidates hard to manipulate? Journal of the ACM, 54 (3), 1-33, 2007.

[12] J. Davies, G. Katsirelos, N. Narodytska and T. Walsh. Complexity of and Algorithms for Borda Manipulation In Proceedings of AAAI-11, pages 657-662, 2011.

[13] B. Dutta and M. Jackson and M. Le Breton. Strategic candidacy and voting procedures. Econometrica, Vol. 69, No. 4, pages 1013-1037, 2001. 
[14] E. Elkind, P. Faliszewski, and A. Slinko. Cloning in elections. In Proceedings of AAAI-10, pages 768$773,2010$.

[15] E. Elkind, P. Faliszewski, and A. M. Slinko. Swap bribery. In Proceedings of SAGT, pages 299-310, 2009.

[16] P. Faliszewski, E. Hemaspaandra and H. Schnoor. Copeland voting: ties matter. AAMAS-08 (2), pages 983-990, 2008

[17] P. Faliszewski, E. Hemaspaandra, and L. Hemaspaandra. Multimode control attacks on elections. In Proceedings of IJCAI-09, pages 128-133, 2009.

[18] P. Faliszewski and A. Procaccia. The AI war on manipulation: are we winning? AI Magazine 31(4): 53-64 (2010).

[19] M. Garey and D. Johnson. Computers and intractability. A guide to the theory of NP-completeness. Freeman, 1979.

[20] N. Hazon, Y. Aumann, S. Kraus, and M. Wooldridge. Evaluation of election outcomes under uncertainty. In Proceedings of AAMAS-08, pages 959-966, 2009.

[21] E. Hemaspaandra, L. Hemaspaandra, and J. Rothe. Anyone but him: The complexity of precluding an alternative. Artificial Intelligence, 171(5-6):255-285, 2007.

[22] K. Konczak and J. Lang. Voting procedures with incomplete preferences. In Proceedings of the IJCAI-05 Multidisciplinary Workshop on Advances in Preference Handling, 2005.

[23] J.-F. Laslier. Aggregation of preferences with a variable set of alternatives. Social Choice and Welfare, 17(2):269-282, 2000.

[24] G. Laffond, J. Lainé and J.-F. Laslier. Composition-consistent tournament solutions and social choice functions. Social Choice and Welfare, 13(1):75-93, 1996.

[25] T. Lu and C. Boutilier. The Unavailable Candidate Model. A Decision-Theoretic View of Social Choice. ACM Conference on Electronic Commerce 2010, pages 263274, 2010.

[26] M.S. Pini, F. Rossi, K. Brent Venable, and T. Walsh. Incompleteness and incomparability in preference aggregation. In Proceedings of IJCAI'07, pages 1464-1469, 2007.

[27] C. R. Plott. Axiomatic social choice theory: an overview and interpretation. American Journal of Political Science, 20:511-596, 1976.

[28] T. Tideman. Independence of clones as a criterion for voting rules. Social Choice and Welfare, 4(3):185206, 1987.

[29] T. Walsh. Complexity of terminating preference elicitation. In Proceedings of AAMAS-08, pages 967974, 2008.

[30] L. Xia and V. Conitzer. Determining Possible and Necessary Winners Given Partial Orders. J. Artif. Intell. Res. (JAIR) 41: 25-67, 2011. 
[31] L. Xia and V. Conitzer. Compilation complexity of common voting rules. In Proceedings of AAAI-10, pages 915-920, 2010.

[32] L. Xia, M. Zuckerman, A. Procaccia, V. Conitzer, and J. Rosenschein. Complexity of unweighted coalitional manipulation under some common voting rules. In Proceedings of IJCAI-09, pages 348-353, 2009.

[33] L. Xia, J. Lang and J. Monnot. Possible winners hen new alternatives join: new results coming up! In Proceedings of AAMAS-11, pages 829-836, 2011. 